\title{
Multidimensional coherent optical spectroscopy of semiconductor nanostructures: a review
}

\author{
Gaël Nardin ${ }^{1,2}$ \\ 1 JILA, University of Colorado \& National Institute of Standards and Technology, \\ Boulder CO 80309-0440, USA \\ ${ }^{2}$ Laboratory of Applied Photonics Devices, School of Engineering, Ecole \\ Polytechnique Fédérale de Lausanne (EPFL), CH - 1015 Lausanne, Switzerland \\ E-mail: gael.nardin@a3.epfl.ch
}

\begin{abstract}
Multidimensional Coherent optical Spectroscopy (MDCS) is an elegant and versatile tool to measure the ultrafast nonlinear optical response of materials. Of particular interest for semiconductor nanostructures, MDCS enables the separation of homogeneous and inhomogeneous linewidths, reveals the nature of coupling between resonances, and is able to identify the signatures of many-body interactions. As an extension of transient Four-Wave Mixing (FWM) experiments, MDCS can be implemented in various geometries, in which different strategies can be used to isolate the FWM signal and measure its phase. I review and compare different practical implementations of MDCS experiments adapted to the study of semiconductor materials. The power of MDCS is illustrated by discussing experimental results obtained on semiconductor nanostructures such as quantum dots, quantum wells, microcavities, and layered semiconductors.
\end{abstract}

Keywords: Fourier transform spectroscopy, Four-Wave-Mixing, Excitons, Biexcitons, Quantum Wells, Quantum Dots

\section{Introduction}

Optical spectroscopy is one of the most essential tool to study semiconductor materials. Using rather simple techniques such as linear absorption or photoluminescence spectroscopy, physicists are able to reveal some fundamental properties of semiconductor materials. Of particular interest for optoelectronic devices, these linear methods provide information about the optical properties of the semiconductors, such as the value of the band gap, or the existence of excitons (below-gap bound states made of Coulomb-correlated electron-hole pairs). There are questions, however, that are difficult or impossible to address using simple linear spectroscopy techniques. A first one arises from the situation, very frequent in semiconductor nanostructures, of inhomogeneous broadening. Interface fluctuations in a quantum well (QW) or 
inhomogeneous size distribution within a quantum dot (QD) ensemble provide an inhomogeneous distribution of transition energies. This distribution usually dominate the spectral linewidth, preventing access to the intrinsic, homogeneous linewidth of a resonance. Another topic of interest is the occurrence of coupling between semiconductor nanostructures. The coupling between electrons and holes confined in separate QWs or QDs can be exploited for various applications such as quantum cascade lasers [1] or in the perspective of implementing multi-bit quantum information processors [2]. To measure homogeneous linewidths or assess coupling between nanostructures, nonlinear or time-resolved techniques are needed. This paper reviews Multidimensional Coherent optical Spectroscopy (MDCS) techniques and show how they can measure the coherent, ultrafast nonlinear optical response of semiconductor nanostructures. It will be shown how MDCS can elegantly separate homogeneous and inhomogeneous broadening, assess coupling between excitonic transitions, and reveal the role of many-body interactions in the nonlinear response of optical excitations $\ddagger$.

In Section 2, I show how MDCS, which is an extension of Four-Wave Mixing (FWM) spectroscopy, can assess the nonlinear light-matter interaction, in a perturbative limit up to the third order. In particular, I introduce double-sided Feynman diagrams, which provide an intuitive understanding of perturbative nonlinear light-matter interaction processes. Then I introduce the reader to different pulse sequences that can be used in MDCS schemes (rephasing, non-rephasing and two-quantum), and how they yield different multidimensional spectra (zero-, one-, and two-quantum two-dimensional spectra, and three-dimensional spectra).

In Section 3, I review different ways in which MDCS can be practically implemented. In particular, as a coherent experiment, MDCS needs phase resolution of the FWM signal, and also a scheme to maintain phase stability between the excitation pulses. I will underline a requirement that is specific to semiconductor materials: the need of maintaining phase stability for relatively long delays between the excitation pulses, so that coherence and population times of up to nanoseconds can be probed. An essential step in FWM and MDCS experiments is the isolation of the FWM signal from other nonlinear and linear contributions. I will describe methods based on momentum conservation that are implemented in non-collinear geometries, with several possible variations. Then I will introduce other signal isolation methods (phase cycling, frequency selection), which can be used in addition to the momentum-conservation-based methods, or on their own in a collinear geometry with the detection of a population signal. I will also underline the link between dimensionality of the sample and geometry, and show how collinear geometry experiments can be used to measure FWM and perform MDCS of low-dimensional nanostructures.

In Section 4, I present results, obtained in various research groups, that demonstrate the ability of MDCS to clarify congested spectra and reveal the fundamental properties of semiconductor nanostructures. It will be shown how MDCS enables separation and

$\ddagger$ Selected contributions from the author's own group were recently reviewed in Ref. [3] 
measurement of homogeneous and inhomogeneous linewidths in disordered quantum wells, large ensemble of quantum dots and emerging semiconductor materials, and how this can be useful for measuring biexciton binding energies. I also focus on one of the major advantages of MDCS, which is the ability to study coupling between separate resonances. I provide examples of the coupling between heavy hole and light hole excitons in GaAs quantum wells, and between excitons confined in separate QWs and QDs. These examples also illustrate the ability of MDCS to identify the role of manybody interactions in the nonlinear optical response of excitons and exciton-polaritons.

\section{Double-sided Feynman diagrams and types of multidimensional spectra}

Multidimensional coherent optical spectroscopy is essentially a FWM experiment. Here, I briefly describe how the FWM experiment can be described as a perturbative expansion of light-matter interaction up to the third order. I do not pretend to give a rigorous derivation - for which I invite the reader to turn to nonlinear optics textbooks $[4,5]$ - but merely to introduce the tools with which one can read and interpret a multidimensional spectrum. Let us consider the light-matter interaction described by the Hamiltonian $H=H_{0}+H_{i}$, where $H_{0}=\left(\begin{array}{cc}E_{0} & 0 \\ 0 & E_{1}\end{array}\right)$ describes an unperturbed two-level system (ground state $|0\rangle$ and excited state $|1\rangle-$ Fig $3(\mathrm{a}))$ and $H_{i}=-E(t)\left(\begin{array}{cc}0 & \mu \\ \mu & 0\end{array}\right)$ is the interaction with a time-varying field $E(t)$, and $\mu$ is the dipole moment that couples the two-level system to the electromagnetic field. The equation of motion for the density matrix $\rho=|\psi\rangle\langle\psi|$ can be written as $\dot{\rho}=-\frac{i}{\hbar}[H, \rho]$. The equations of motion for the density matrix elements are the so-called Optical Bloch Equations (OBEs), which are able to describe the non-perturbative phenomenon of Rabi flopping [5].

On the contrary, MDCS considers weak fields for which the light-matter interaction can be treated perturbatively. The field of excitation pulses can be expressed as $E(t)=\tilde{E}(t) e^{-i(\omega t-\vec{k} . \vec{r})}+$ c.c., where $\tilde{E}(t)$ is the pulse envelope, $\omega$ is the centre optical frequency, and $\vec{k}$ is the momentum. As will be detailed in Section 3, the experimental conditions determine whether $\tilde{E}(t) e^{-i(\omega t-\vec{k} \cdot \vec{r})}$ or its complex conjugate contributes to the nonlinear signal of interest. In the perturbative limit, a pulse $A$ will induce a coherence - or polarization - in the two-level system (i.e. a coherent superposition between the ground and excited states). In the density matrix formalism, the system is driven from the ground state population $\rho_{00}=|0\rangle\langle 0|$ to the coherence $\rho_{10}=|1\rangle\langle 0|$. Similarly, an action of pulse $A$ and its complex conjugate $A^{*}$ can create an excited state population: $\rho_{00} \stackrel{A}{\rightarrow} \rho_{10} \stackrel{A^{*}}{\rightarrow} \rho_{11}$. The creation of a first order polarization in the two-level system by a single pulse also yields a radiated field - the free induction decay. The linear response function associated with this phenomenon can be associated to a double-sided Feynman diagram [4] describing the temporal evolution of the density matrix (Fig. 1).

The free induction decay of the coherence $|1\rangle\langle 0|$ destructively interferes with the transmitted part of the excitation field to produce the well-known absorption dip in a 


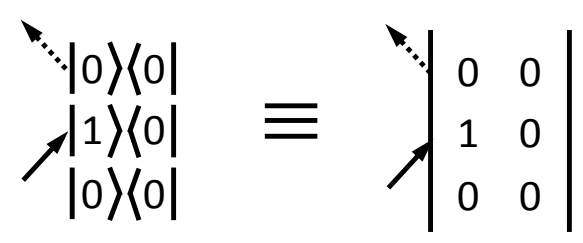

Figure 1. Example of double-sided Feynman diagram, describing the time evolution of the density matrix. The process shown here describes the linear response of a twolevel system. The solid arrow indicate interaction with an incident field. The dashed arrow indicates a radiated field. Increasing time is going upward. Inward arrows indicate excitation, outward arrows indicate de-excitation. In the common notation (right-hand side), bra and ket are simplified.

transmission spectrum.
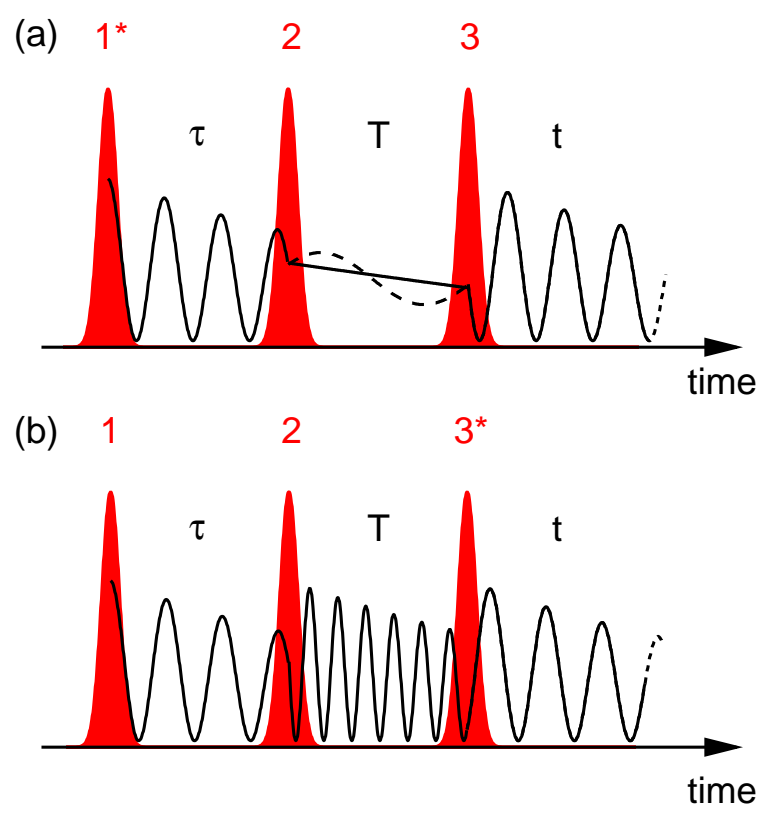

Figure 2. Typical pulse sequences used in MDCS. Pulse sequence $(1,2,3)$, with inter-pulse delays $\tau$ and $T . t$ is the real time during which the radiation of the FWM signal occurs. * indicates that the conjugated part of the excitation pulse is acting. (a) Rephasing $\left(S_{I}\right)$ sequence. A first order coherence is induced by the first pulse, evolving during $\tau$. After pulse 2, a population decay (solid line) and/or a Raman coherence (dashed line) occur during $T$. After pulse 3, a third-order coherence is induced, and radiates as the FWM signal. (b) $S_{I I I}$ sequence, characterised by the two-quantum coherence induced after the second pulse.

These concepts can be extended to nonlinear spectroscopy, in particular FWM experiments, where nonlinear response functions can be associated with similar doublesided Feynman diagrams, each of them representing a particular quantum pathway. In a FWM experiment involving three incident fields and one radiated field, I label the excitation pulses 1, 2, and 3, with inter-pulse delays $\tau$ and $T$ (Fig. 2). $t$ is the real time, after pulse 3 , during which the FWM signal is radiated. 


\subsection{One-quantum rephasing and non-rephasing $2 D$ spectra}

The three-pulse sequences that induce a FWM signal in a two-level system include one of the pulses acting as conjugate. A pulse sequence where the first pulse is conjugate, as shown in Fig 2 (a) is referred to as a $S_{I}$ sequence. The corresponding possible Feynman diagram are shown in Fig. 3 (b). These diagrams show that the coherence $\rho_{01}$ induced by pulse 1 has a phase evolution opposite to the coherence $\rho_{10}$ induced after pulse 3 . This phase evolution in opposite directions is the origin of the well-known photon echo in the presence of an inhomogeneous distribution of transitions [6]. The echo occurs at time $t=\tau$ when the phase evolution during time $t$ cancels that of time $\tau$, bringing the inhomogeneous distribution of oscillators in phase again and yielding the radiation of a macroscopic signal. The $S_{I}$ sequence, and its associated Feynman diagrams are thus referred to as rephasing. The Feynman diagrams of the pulse sequence $S_{I I}$, in which the conjugated pulse is the second, are shown in Fig 3 (c). In this case, the phase of the coherence $\rho_{10}$ during time $\tau$ evolves in the same direction as during time $t$. No echo is produced in this case, and this sequence is thus referred to as non-rephasing. For a given value of delays $\tau$ and $T$, the radiated field can be spectrally resolved along emission frequency $\omega_{t}$ (details are given in Section 3). Stepping delay $\tau$ and performing a Fourier transform with respect to this delay, the data can be displayed as a $2 \mathrm{D}$ spectrum, with absorption and emission frequency axes $\omega_{\tau}$ and $\omega_{t}$, as shown for a single two-level system (homogeneous limit) in Fig. 4 (a).

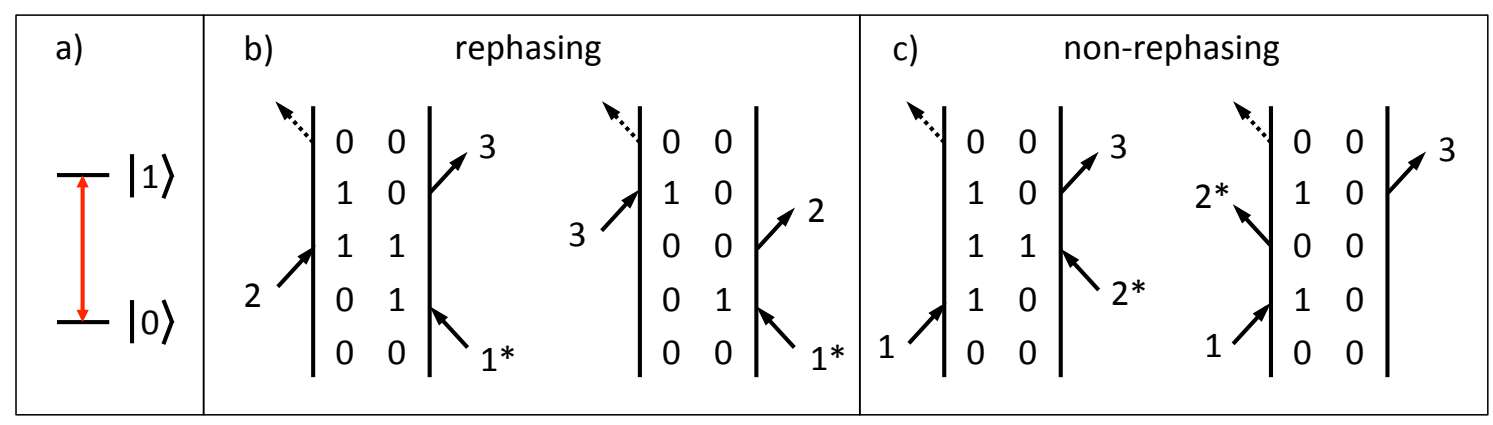

Figure 3. (a) Two-level system with an optically allowed transition from ground state $|0\rangle$ to excited state $|1\rangle$. (b) Double-sided Feynman diagrams representing rephasing quantum pathways for a two-level system. * indicate that the first pulse acts as conjugated. (b) Same for non-rephasing pathways, where the second pulse acts as conjugated.

\subsection{Inhomogeneous broadening in rephasing $2 D$ spectra}

A $2 \mathrm{D}$ scan recorded with a $S_{I}$ pulse sequence provides access to the homogeneous linewidth (or, in other words, the intrinsic dephasing) of a resonance, even within an inhomogeneous distribution. Figure 4 shows calculations based on OBEs for different quantities of inhomogeneous broadening (adapted from [7]). In Fig. 4 (a) is shown the $2 \mathrm{D}$ spectrum expected for a single two-level system, i.e. the homogeneous limit. In this 


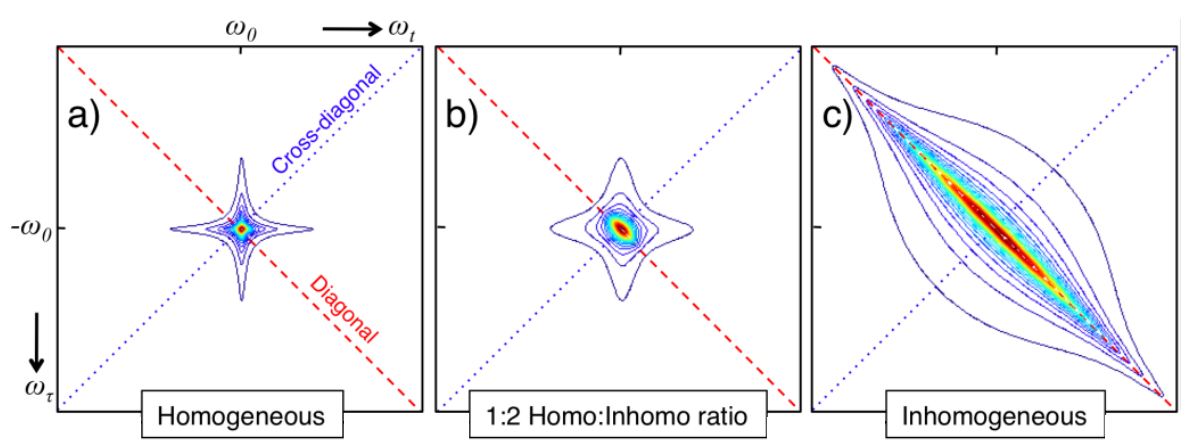

Figure 4. Absolute value of a calculated 2D rephasing spectrum, for a homogeneous transition (a), a slightly inhomogeneously broadened transition (b) and in the limit of a very inhomogeneous ensemble of transitions (c). Adapted from Ref. [7].

case, a single peak is obtained at the resonance frequency $\omega_{0}$. The $\omega_{\tau}$ frequency axis is plotted as negative, because the coherence induced by the conjugate first pulse during $\tau$ evolves in the direction opposed to the one during $t$. In 4 (b), the case of a slightly inhomogeneous distribution of oscillators is shown. The photon echo that would appear in the time domain translates itself as an elongated peak along the diagonal $\omega_{\tau}=\omega_{t}$ in the spectral domain. This effect is even more pronounced in the limit of a very inhomogeneous distribution (Fig. 4 (c)). In this case, the inhomogeneous distribution of oscillators is clearly spread along the diagonal, while the homogeneous linewidth can be measured along the cross-diagonal direction. Analytical line shapes have also been derived for the intermediate case shown in Fig. 4 (b) [7]. These spectra enable a clear and straightforward visualisation, separation and measurement of homogeneous and inhomogeneous linewidths. While time-domain photon-echo spectroscopy also enables the measurement of homogenous linewidth (or dephasing rate) within inhomogeneous ensembles [8], the rephasing 2D spectrum enables the measurement of these quantities as a function of emission or absorption energies. Examples of the advantages provided by this feature will be given in Section 4.3, e.g. the measurement of dephasing rate as a function of QD size within an ensemble.

\subsection{Two resonances in rephasing $2 D$ spectra}

The rephasing 2D spectrum can also provide useful information in the presence of two resonances $\omega_{1}$ and $\omega_{1^{\prime}}$. If the two resonances are uncoupled, coherently coupled via a common ground state (forming a three-level $V$ system), coupled through a population transfer, or forming a diamond-shaped 4-level system, different Feynman diagrams can be written, corresponding to different contributions in rephasing $2 \mathrm{D}$ spectra. In the first case of two uncoupled transitions (Fig. 5 (a)), each transition can simply be associated with the Feynman diagrams of a single two-level system. This results in a 2D rephasing spectrum with two peaks on the diagonal, as shown in the schematic spectrum of Fig. 6 (a). In the case of two transitions forming a $V$-shape three-level system, new quantum pathways are available, whose diagrams are shown in Fig. 5 (b). These diagrams are a 
signature of the coherent coupling through the shared ground state and correspond to off-diagonal peaks (or cross peaks), as shown in Fig. 6 (b). A third possibility arises from the population transfer (e.g. a relaxation from the upper to the lower energy transition) during delay $T$ (Fig. 5 (c)). This relaxation is qualified as incoherent, since there is no phase evolution during population time. This contribution also provides a cross peak in the rephasing 2D spectrum, shown in Fig. 6 (c) - a lower cross peak for relaxation, an upper cross peak for activation. The three $2 \mathrm{D}$ spectra of Fig. 6 show distinct features in the cases of uncoupled, coherently coupled and incoherently coupled transitions, providing a direct, unambiguous distinction between quantum beats and polarization interferences observed in FWM experiments [9]. Another way to model coherent coupling between the two transitions is with the diamond shape four-level system (Fig. $5(\mathrm{~d})$ ). While a symmetric diamond system is equivalent to two uncoupled transitions, the introduction of an energy shift $\Delta E$ (interaction energy) of the mixed twoquantum state couples the transitions [10]. Explicitly, new Feynman diagrams including the upper transitions can be written, corresponding to cross peak contributions (e.g. diagrams for the lower cross peak are given in Fig. $5(\mathrm{~d})$ ). In the absence of interaction energy $\Delta E=0$ these new pathways exactly cancel with those of the three-level system, resulting in no cross peak in the $2 \mathrm{D}$ spectrum. For a non-zero $\Delta E$, positive and negative contributions are shifted on the emission energy axis, providing a cross peak. When the real part of the $2 \mathrm{D}$ spectrum is plotted instead of only its absolute value, the opposite sign of the two frequency shifted contributions results in a clear dispersive line shape. Examples will be given in Section 4 where the interaction energy can be associated with two-exciton interactions. Other examples will show the situation when interaction energy is larger than the linewidth (e.g. biexciton binding energy), in which case the two contributions give rise to two separate peaks.

\subsection{Zero-quantum $2 D$ spectra and $T$ dependence}

Within a $S_{I}$ or $S_{I I}$ pulse sequence, it is also possible to step the delay $T$, during which the dynamics of population decay, population transfer and/or non-radiative coherences can be observed. When $T$ is stepped instead of $\tau$, a Fourier transform provides a zeroquantum 2D spectrum, plotted as a function of $\omega_{T}$ and $\omega_{t}$ for a fixed delay $\tau$ [12]. In such a spectrum, population terms contribute to a zero-energy peak, whose linewidth is characteristic of population decay rates. In coherently coupled systems such as the three-level $V$ system or the four-level 'diamond' system, coherent superpositions between excited states can be induced during $T$ (e.g. $\rho_{1^{\prime} 1}$ in the second diagram of Fig. 5 (b)). This coherence will evolve at the difference frequency between the two transition energies (generally far from the visible spectrum, and thus sometimes referred to as a non-radiative or Raman coherence). This non-radiative coherence will appear as a non-zero-energy peak (at its characteristic difference frequency) in the zero-quantum spectrum. Alternatively, delay $T$ can be stepped in addition to $\tau$, so as to follow the evolution of the different peaks in a $\left(\omega_{\tau}, \omega_{t}\right)$ spectrum as a function of $T$. In this way, 


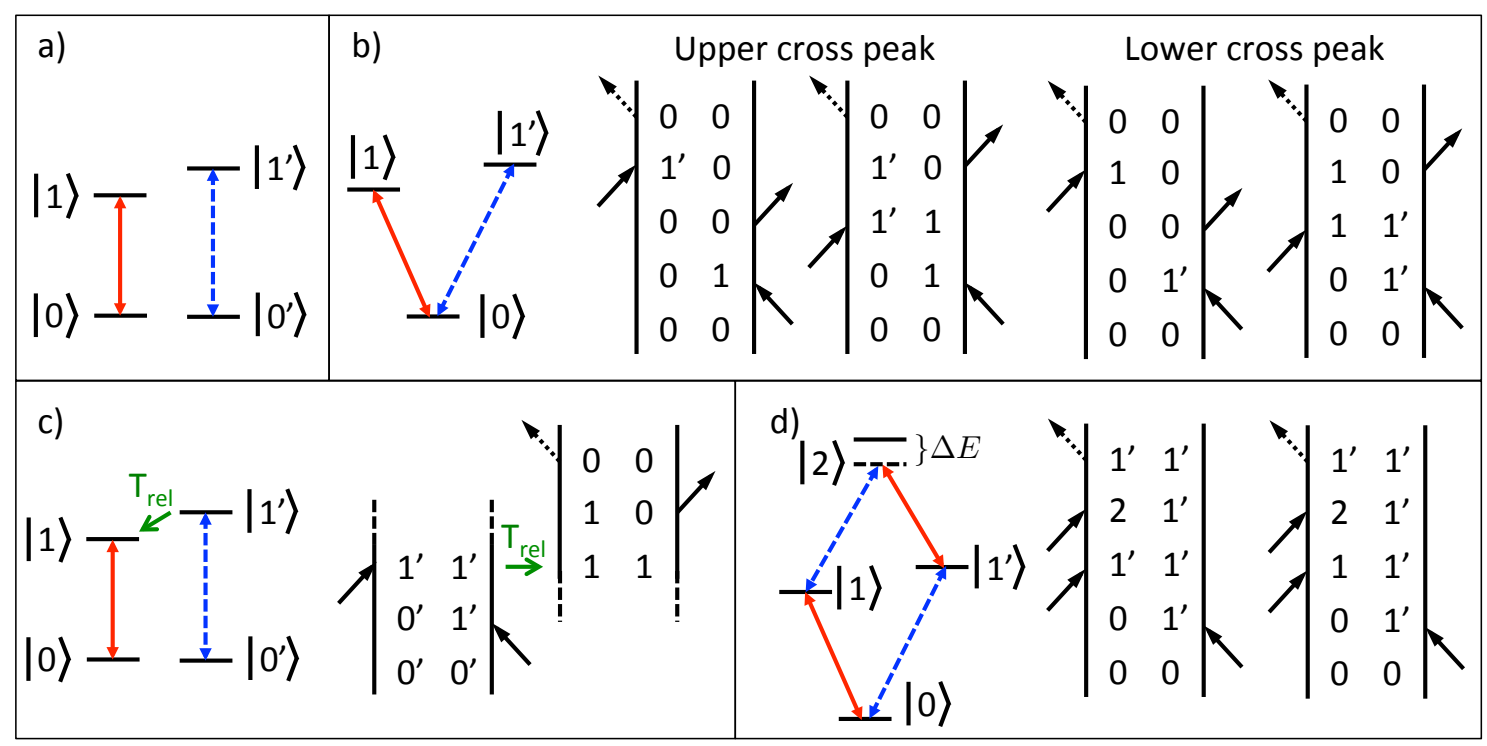

Figure 5. (a) Two uncoupled two-level systems. (b) A three-level 'V' system, where the two transitions are coupled through a common ground state. Feynman diagrams are shown for the rephasing quantum pathways resulting in upper and lower crosspeaks. (c) Two two-level systems are coupled incoherently via a relaxation from the high-energy transition to the low-energy transition. (d) Four-level 'diamond' system, representing two two-level systems coupled via a mixed two-quantum state. $\Delta E$ is the interaction energy (e.g. biexciton binding energy). The two-quantum-state gives rise to four new quantum pathways (only two diagrams, contributing to the lower cross peak, are shown as examples) that can be achieved with a rephasing $\left(S_{I}\right)$ pulse sequence.
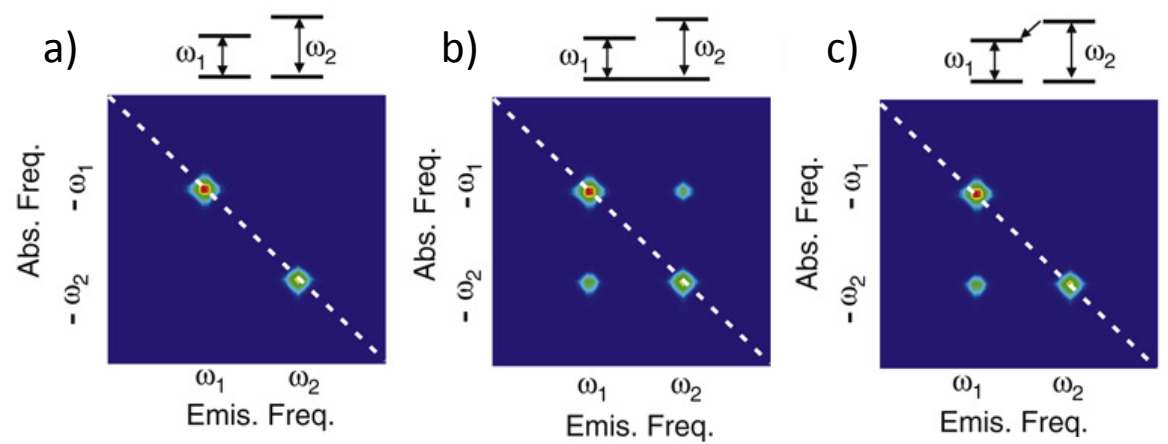

Figure 6. Schematic 2D rephasing spectra for the cases of (a) two uncoupled transitions, (b) two transitions coupled via a shared ground state in a three-level 'V' system, and (c) Two two-level systems coupled via an incoherent population relaxation. Adapted from Ref. [11] .

population transfers (as depicted in Fig. 5 (c)) can be distinguished from population decay. Examples will be given in Section 4 for the study of incoherent and coherent coupling within QWs and QDs and to measure the fine-structure splitting in QDs. 


\subsection{Two-quantum 2D spectra in a three-level ladder system}

I have introduced so far $S_{I}$ (rephasing) and $S_{I I}$ (non-rephasing) pulse sequences. Another one is the $S_{I I I}$ sequence, in which the conjugate pulse comes third. This sequence induces two-quantum coherences (i.e. a coherence between ground state and second excited state) in systems characterised by a three-level ladder, as in Fig. 7. With this pulse sequence, the second rung of the ladder is reached without going through a population state, as was demonstrated with the biexciton level in GaAs QWs [13]. Taking a Fourier transform of the data with respect to $T$ provides a two-quantum $2 \mathrm{D}$ spectrum as a function of the two-quantum energy $\hbar \omega_{T}$ and the emission energy $\omega_{t}$ $[14,15]$. Similarly to what was discussed with the four-level diamond system, the twoquantum pathways of a three-level ladder system cancel in the absence of interaction energy (Fig. 7). Two-quantum 2D spectra are thus extremely sensitive to interactions and many-body effects [14], as will be shown with examples involving QW exciton many-body coherences in Section 4.

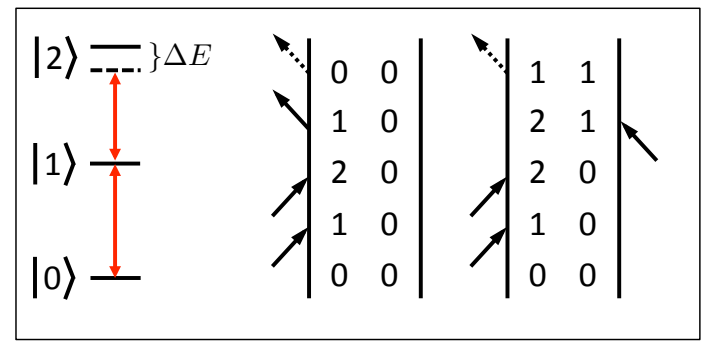

Figure 7. A three-level 'ladder' system. The upper transition is shifted by $\Delta E$, the two-quantum (e.g. two-exciton) interaction energy. Two Feynman diagrams are shown, where a two-quantum coherence $\rho_{20}$ is induced after excitation by the two first pulses of a $S_{I I I}$ sequence.

\subsection{D spectroscopy}

Three-dimensional spectroscopy [16] is the extension of 2D spectroscopy that is achieved when stepping delays $\tau$ and $T$. When phase stability between all pulses is ensured, Fourier transforms enable the visualisation of the data as a $3 \mathrm{D}$ spectrum $\left(\omega_{\tau}, \omega_{T}, \omega_{t}\right)$. The 3D spectrum provides at the same time distinction between diagonal and offdiagonal peaks, and between population and coherence terms. It can be sliced in any direction to produce the 2D spectra described previously (examples are available with the light hole / heavy hole exciton system in single QW [17] and in coupled QWs [18, 19]). Taken as a whole, the 3D spectrum provides a complete picture of the nonlinear lightmatter interaction up to the third order, unraveling quantum pathways to ultimately "measure" the Hamiltonian [20]. 


\section{Experimental Methods}

In this section, I discuss various implementations of MDCS. The concept of MDCS and Fourier transform spectroscopy has originally been developed in the field of Nuclear Magnetic Resonance (NMR) spectroscopy [21], where nuclear spins are nonperturbatively driven by radio frequency fields. Thanks to advances in the generation of femtosecond laser pulses, chemists brought multidimensional Fourier transform to the IR [22] and visible [23] domains to study ultrafast molecular vibrational and electronic coherences, respectively. Let us also note that Fourier transform (or timedomain) spectroscopy is routinely used in the $\mathrm{THz}$ domain [24, 25], where grating-based spectrometers and cameras are not readily available. 2D $\mathrm{THz}$ spectroscopy has been demonstrated on inter-subband excitonic transitions in QW structures using electrooptic sampling in a collinear geometry $[26,27,28]$. In the following, we will focus on the experimental methods that address inter-band transitions in the optical (visible) domain - sometimes referred to as electronic spectroscopy since it probes the electronic degrees of freedom, as opposed to vibrational spectroscopy. The challenge in this case resides in the stabilisation of inter-pulse delays with interferometric precision. Emphasis will also be given to methods that enable sufficiently large inter-pulse delays to be achieved, so that the potentially long coherence and population times of epitaxially grown semiconductor nanostructures can be probed - up to nanosecond time scales. The section sorts possible implementations of MDCS based on their geometry (non-collinear or collinear), which is linked to different ways of isolating the nonlinear signal of interest (based on momentum conservation, phase-cycling, or selection in the frequency domain) and to various methods of ensuring stable inter-pulse delays (passively or actively).

Let us note that it is also possible to produce $2 \mathrm{D}$ spectra by spectrally resolving a photoluminescence excitation signal (i.e. recording a photoluminescence spectrum for different values of a continuous wave or narrow band excitation wavelength), - see e.g. [29], or using a two-colour pump-probe experiment - see e.g. [30]. While comparison with simulations can provide some information on coherent coupling mechanisms [30], these experiments do not, in general, provide a straightforward access to coherent processes, as only incoherent contributions (relaxation or thermal activation) appear as cross-peaks in the $2 \mathrm{D}$ spectrum.

\subsection{Isolation of FWM using conservation of momentum}

The most widely used way to isolate a FWM signal of interest from other linear and nonlinear contributions relies on the conservation of momentum, in a non-collinear geometry. For three excitation pulses incident on a sample with wave vectors $\vec{k}_{1}, \vec{k}_{2}$ and $\vec{k}_{3}$ (in chronological order), FWM signals are emitted in a phase-matched direction given by momentum conservation. In particular, directions given by $-\overrightarrow{k_{1}}+\overrightarrow{k_{2}}+\overrightarrow{k_{3}}$, $\overrightarrow{k_{1}}-\overrightarrow{k_{2}}+\overrightarrow{k_{3}}$ and $\overrightarrow{k_{1}}+\overrightarrow{k_{2}}-\overrightarrow{k_{3}}$ enable isolation of rephasing $\left(S_{I}\right)$, non-rephasing $\left(S_{I I}\right)$, and two-quantum $\left(S_{I I I}\right)$ signals, respectively. The most common non-collinear geometries are depicted in Fig. 8. 


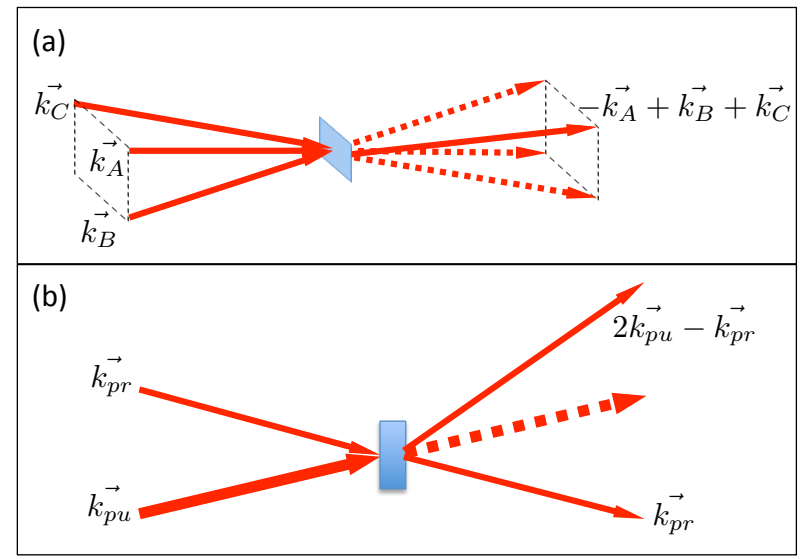

Figure 8. Most common implementations of MDCS in non-collinear geometries, where the FWM signal of interest is isolated thanks to momentum conservation rules. (a) 'Box' geometry, where the FWM signal is detected in the $\vec{k}_{F W M}=-\overrightarrow{k_{A}}+\overrightarrow{k_{B}}+\overrightarrow{k_{C}}$ direction. (b) 'Pump-probe' geometry. Thick (thin) input arrows depict the pump (probe). Plain output arrows show directions $\vec{k}_{F W M}=2 \overrightarrow{k_{p u}}-\overrightarrow{k_{p r}}$ and $\vec{k}_{F W M}=$ $\overrightarrow{k_{p u}}-\overrightarrow{k_{p u}}+\overrightarrow{k_{p r}}=\overrightarrow{k_{p r}}$, separated from the transmitted pump (dashed arrow).

3.1.1. Box geometry Although not the most straightforward to implement, the 'box' geometry [15, 31, 32, 33, 34, 35] (Fig. 8 (a)) is versatile and conceptually easy to understand. The three excitation beams $A, B$ and $C$ are focused on the sample with three distinct directions (corresponding to the three corners of a square - the box - in the momentum space), and the FWM signal is selected in the phase-matched direction $\vec{k}_{F W M}=-\overrightarrow{k_{A}}+\overrightarrow{k_{B}}+\overrightarrow{k_{C}}$ (corresponding to the fourth corner of the square). This direction ensures that pulse $A$ is acting as the conjugate pulse in the sequence. Rephasing, nonrephasing or two-quantum signals can be selectively obtained by having the conjugated pulse $A^{*}$ arriving on the sample in the first, second or third position [15, 34, 35]. The signal is spectrally resolved by a grating-based spectrometer on a CCD camera - providing, in a single acquisition, the Fourier transform of the signal with respect to emission time $t$. Phase resolution is obtained through spectral interferometry [36], where the signal is heterodyned with a reference pulse (local oscillator (LO)). The LO can either be routed around the sample [33, 34] or through the sample [31, 35], in which case the amplitude and phase distortion of the LO needs to be accounted and compensated for [37]. Based on a similar principle, the use of the box geometry in a reflection geometry has also been demonstrated [38], and can be used in the case of optically dense samples.

To obtain the additional spectral dimensions, inter-pulse delay $\tau(T)$ is stepped and a phase-resolved spectrum is recorded for every step. Then, the data set is Fourier transformed with respect to $\tau(T)$ to generate the absorption (mixing) frequency axes $\omega_{\tau}\left(\omega_{T}\right)$. The main challenge of optical MDCS resides in the phase stabilization during each spectrum acquisition and between the spectra recorded at every $\tau$ and $T$ step, in order to obtain a reliable Fourier transform spectrum. In other words, a control of the inter-pulse delays needs to be achieved with an accuracy much smaller than the 
optical wavelength. A clever way to obtain intrinsic phase stability is by generating the excitation and reference pulses using diffractive optics [32, 35, 39]. In this way, all excitation and reference beams travel through the same optical elements, ensuring an optimal passive stabilization of inter-pulse delays. Reference [35] describes the Coherent Optical Laser Beam Recombination Technique (COLBERT) spectrometer: a versatile configuration where the non-collinear beam geometry is generated by diffracting on a spatial light modulator (SLM), and then phase pulse-shaping involving a second SLM provides delays between the pulses. This method offers several advantages, such as the straightforward implementation of phase-cycling algorithms [40] without any modification of the apparatus for additional isolation of the FWM signal from linear scattering contributions (phase-cycling will be described more in details in Section 3.2). Combining phase and diffraction-based amplitude pulse shaping [41], COLBERTtype experiments can also independently shape the spectrum of every pulse, a method that enables further isolation of contributions to the nonlinear signal through pathwayselective excitation [42]. All these features are achieved without any moving part in the setup. This method has been successfully applied to record multidimensional spectra of semiconductor quantum wells (QWs) [17, 18, 19, 35].

The main limitation of pulse-shaping-based coherent spectroscopy is the achievable inter-pulse delays, limited by the resolution of the SLM to the order of 10ps [35]. Consequently, such experiments are not able to probe population and coherence times of semiconductor nanostructures such as QWs (coherence times of ten's of picoseconds [8]) and QDs (coherence times up to nanoseconds [43, 44]). This translates into the inability to measure homogeneous linewidths of these nanostructures, since a delay of 10 ps corresponds to a resolution limit of about half a meV for the $\omega_{\tau}$ and $\omega_{T}$ axes.

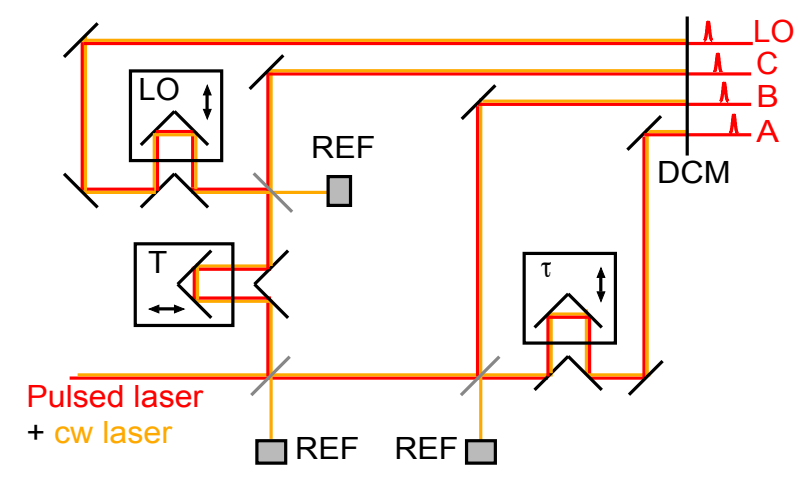

Figure 9. Scheme of actively stabilised, mechanical delay line-based generation of four non-collinear pulses - 3 excitation, one local oscillator (LO). A $c w$ reference laser runs through the setup, and is reflected back using a dichroic mirror (DCM) to form three nested Michelson interferometers. Stabilization of inter-pulse delays is ensured by monitoring the reference laser interference on reference detectors (REF) [33, 34].

In order to capture the long coherence and population times associated with semiconductor materials, mechanical delay stages are required. To maintain phase stability over these long delays, active stabilization schemes have been implemented 
$[33,34,45]$. Actively stabilised MDCS is based on a reference continuous wave (cw) laser running through the delay stages, and reflecting on a dichroic mirror to form Michelson interferometers (Fig. 9). The interference of the $\mathrm{cw}$ laser is then monitored on reference detectors for every delay stage independently, and feeds an electronic feedback loop to control piezo-electric actuators that adjust the inter-pulse delays with interferometric precision. A particularly successful example is the Multidimensional Optical Nonlinear Spectrometer (MONSTR) experiment [34, 46], combining active feedback with an ultrastable platform. The MONSTR has been able to capture coherence time and measure homogeneous linewidths of semiconductor QWs [7, 47] and QD ensembles [48, 49]. In this kind of experiment where mechanical delay lines are used, liquid crystal retarders can be added to implement phase-cycling algorithms as a way to further isolate the FWM signal from other contributions [34].

In addition to rephasing, non-rephasing and two-quantum spectra, correlation (also called purely absorptive) spectra can be obtained in the box geometry as the sum of real parts of rephasing and non-rephasing spectra, measured separately [50]. The versatility of the box geometry comes with the price of a demanding alignment. Also, while active or passive stabilization ensures the correct phasing of the different one-dimensional spectra, there is still an unknown general phase offset (the phase between FWM signal and reference), preventing the correct separation of real and imaginary parts of the multidimensional spectra. Additional procedures are needed to determine this offset and separate real and imaginary parts $[33,51,52,53]$. The most common method, a comparison with a phase-resolved pump-probe spectrum mimicking the FWM signal at zero delay $\tau$ [33], is not compatible with $S_{I I I}$ scans nor cross-polarised pulse sequences.

Let us note that, although the non-collinear geometries enables isolation of a FWM signal in a particular momentum-conserving direction, higher-order signals can also radiate into that direction. For example, a fifth-order signal involving three interactions with the pulse $A$ can still radiate in the direction $-\overrightarrow{k_{A}}+\overrightarrow{k_{A}}-\overrightarrow{k_{A}}+\overrightarrow{k_{B}}+\overrightarrow{k_{C}}=-\overrightarrow{k_{A}}+\overrightarrow{k_{B}}+\overrightarrow{k_{C}}$. A power dependence to check that the signal intensity scales with $P^{3}$ (with $P$ the average power in the excitation beams) is needed to ensure that the observed signal is dominated by third-order contributions - and can be used to separate higher-order contributions within a two-dimensional spectrum [49].

3.1.2. Partially collinear geometries (pump-probe) The 'pump-probe' geometry (Fig. 8 (a)) is a partially collinear geometry where two of the three excitation pulses are incident with the same wave vector $\overrightarrow{k_{p u}}$. It inherits its name from the equivalency to a pump-probe experiment in the case $\tau=0$. The pump-probe geometry has the advantage of being relatively easy to build as an extension of an existing pump-probe setup [54], where the pump pulse pairs can be generated using phase-resolved pulse shaping - based either on spatial light modulation [55] or acousto-optical pulse shaping $[56,57]$ - or a birefringent delay line [58]. Using these schemes, the polarization of the two pump pulses is restricted to either co-polarized [55, 56, 57], or cross-polarized [58], 
but swapping from one to the other with the same system is not possible§. Also, it should be noted that if no stabilization of the delay $T$ between pump and probe pulses is provided, then measuring two-quantum $2 \mathrm{D}$ spectra is not possible. As depicted in Fig. 8 (b), the pump-probe geometry enables the selection of different FWM signals, depending on the phase-matching direction chosen.

The first possibility is to detect the FWM signal in the $\overrightarrow{k_{p r}}$ direction, for which the two 'pump' pulses act once each to create a population. The first order coherence can be probed by stepping the delay $\tau$ between the two pump pulses, and the FWM radiated field is heterodyned with the transmitted probe for phase resolution. In this simple configuration, the two pump pulses are undistinguishable. This fact leads to the simultaneous recording of rephasing and non-rephasing signals, which advantageously provides a direct access to 'purely absorptive' spectra (whereas they are obtained as the sum of the correctly phased rephasing and non-rephasing spectra in the box geometry). Phase-cycling can be used to separate rephasing and non-rephasing contributions $[54,60]$.

A second possibility is to detect the FWM signal in the $2 \overrightarrow{k_{p u}}-\overrightarrow{k_{p r}}$ direction, in which case the reference needs to be routed around the sample for heterodyning. This signal direction enables an independent control of the reference intensity for optimising the reference to signal intensity ratio. On the other hand, care needs to be taken so that the phase between signal and reference remains stable. Interestingly, the direction $2 \overrightarrow{k_{p u}}-\overrightarrow{k_{p r}}$ enables the measurement of one-quantum or two-quantum signals, depending on the time ordering between 'pump' and 'probe' pulses [35, 61]. Looking for signal in other directions such as $3 \overrightarrow{k_{p u}}-2 \overrightarrow{k_{p r}}$ provides access to higher-order signal and correlations, as demonstrated with QW excitons [35] or exciton-polaritons [59].

\subsection{Isolation of FWM in a collinear geometry}

While non-collinear geometries can successfully isolate nonlinear signals from extended objects such as QWs or dense ensemble of QDs, they generally fail to address single nano-objects or small ensemble thereof, because momentum conservation does not apply in systems where the translational symmetry is broken. To circumvent this issue, frequency modulation or phase-cycling techniques can be implemented to substitute for angular selection of the FWM signal. In the following I classify the experiments into two categories. In the first one, heterodyne spectral interferometry (HSI) is used to detect a radiated field in a collinear pump-probe configuration. The second category of experiments is based on the detection of a population signal.

3.2.1. Collinear pump-probe experiments Isolation of the FWM signal in a collinear pump-probe experiment can be achieved in the frequency domain. The idea was

$\S$ However, the selection of cross-polarized pathways using co-polarized pump pulses was demonstrated in Ref. [59] 
originally developed to measure FWM signal from a semiconductor waveguide [62], a typical structure where one cannot rely on momentum conservation to isolate FWM. In this experiment, acousto-optic modulators (AOMs) were used to frequency-shift reference and probe beams, and the signal was detected at the difference frequency. Extending the idea to 2D spectroscopy, heterodyne spectral interferometry (HSI) [63] was developed to address individual quantum systems such as single QDs [64]. After a frequency shifting by AOMs of the pump and probe beams, this clever scheme uses an additional AOM to mix the output beams and isolate in the frequency domain the interference term of FWM and reference beams. An advantage of the collinear geometry is that it enables the use of a microscope objective for sample excitation and signal collection in a reflection geometry. The microscope objective provides a high spatial resolution, which can be further enhanced by the nonlinear nature of the signal [65]. This apparatus has been used to map [63] and assess [65] coupling between excitons confined in separated QDs (these results will be discussed further in Section 4). Let us note that HSI can also be implemented in addition to a non-collinear pump-probe experiment to enhance sensitivity of the FWM detection in case of strong scattering by the pump beam, as demonstrated with exciton-polaritons in a transmission geometry $[61,66,67]$. A drawback of HSI experiments is that the phase between the pump and probe pulses (and of the reference if the latter is routed around the sample) is not actively stabilised. Spurious phase fluctuations require the use of post-processing and a reference transition to generate the multidimensional spectra, as described in Section 3.2 .2 .

3.2.2. Phase-correction and phase-retrieval algorithms MDCS experiments require, in general, phase stability, so that the Fourier transformation with respect to time delays can be correctly preformed. For example, in experiments where the reference is routed around the sample and no active stabilization is used, unpredictable phase fluctuations between reference and FWM beams result in fluctuations of the spectral phase between individual 1D spectra. This issue can be circumvented by monitoring the phase of a two-level system of reference (e.g. a QD that is assumed to be isolated $[65,68]$ ) and post processing the data to revert the phase changes that occurred due to fluctuations of the optical path. It is thus difficult to use such a method when an isolated two-level system of reference is not available\|. Phase fluctuations can also be addressed during post-processing of the data, using algorithms inspired from the phase-retrieval methods of frequency-resolved optical gating [70]. The convergence of phase-retrieval algorithms has been shown to improve in the case of 3D spectral data [18].

3.2.3. Detecting a population signal All the experiments reviewed so far - collinear or non-collinear - are based on the detection and heterodyning of a radiated field. Another category of experiments rely on the detection of a population signal. Instead of having a

|| A very recent publication shows that qualitative 2D spectra of exciton-polaritons can be still obtained using this method [69]. 


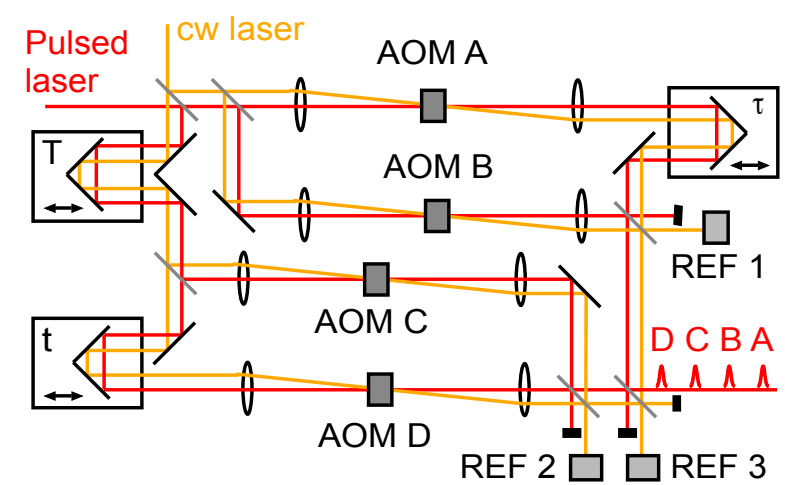

Figure 10. Scheme of a setup to generate four collinear pulses. Individual acoustooptical modulation of each excitation beam enables isolation of the nonlinear signal in the frequency domain. A reference $c w$ laser goes through the same optics, offset from the excitation beam. Detected on reference detectors (REF), the $c w$ laser interference serves as a reference for the lock-in detection of a population signal [71].

FWM process involving three incident fields and a radiated one, these experiments use a fourth incident field to convert the third-order polarization into a fourth-order population. In this case, all spectral axes are generated by Fourier transformation of the inter-pulse delays $\tau, T$ or $t$, without using a conventional, grating-based spectrometer. This concept was first developed and implemented by Tian et al, where the population signal was detected in the form of fluorescence from a Rb atom gas [40]. Since the FWM population signal cannot be isolated in a phase-matched direction, Tian et al implemented phase-cycling algorithms, in analogy with 2D NMR experiments, to separate the FWM signal of interest from other linear and nonlinear contributions, and generate 2D spectra. Phase-cycling relies on the direct dependence of the FWM signal on the phases of the incident pulses. Cycling the phase of the incident pulses can easily be achieved using liquid crystal modulators. Combining the population signal recorded with different input phases enables selection of the nonlinear signal (e.g. rephasing), while contributions from single pulses cancel [72]. Let us note that phase-cycling can also be implemented in addition to wave vector selection in non-collinear configurations $[35,34]$ to suppress contribution from scattering by single pulses on sample inhomogeneities.

Another interesting realisation of phase-cycling in collinear geometry is provided by Aeschlimann et al, where the population signal was measured in the form of photoelectron emission [73]. Using a photo-electron emission microscope apparatus as a detector, the authors were able to measure FWM signal and record 2D spectra from a corrugated silver surface, with a spatial resolution given by the resolution of the electron microscope. Beating the diffraction limit imposed by the wavelength of the excitation pulses, they established coherent multidimensional nanoscopy.

Alternatively, the population signal in a collinear geometry can be isolated in the 
frequency domain. The first realisation of this principle was demonstrated by Tekavec et al, who measured 2D spectra of an atomic $\mathrm{Rb}$ vapour [74]. The apparatus used in this experiment generates a collinear train of four excitation pulses. The setup consists of three nested Mach-Zehnder interferometers, and uses four AOMs to imprint a unique frequency shift on each of the excitation beams. Analogously to wavevector selection in a non-collinear geometry, a filtering in the frequency domain enables isolation of the different nonlinear signals of interest (rephasing, non-rephasing, or two-quantum). This method can be seen as a dynamical way of phase cycling - the AOMs performing a phase modulation on the individual input beams [71]. Signal isolation can be advantageously performed by lock-in amplification. Interestingly, the lock-in reference can be generated such as to reduce the impact of mechanical fluctuations in the setup, providing enough phase stability for the Fourier transformation, without the need of active stabilisation [75]. In the implementation of Tekavec et al, the lock-in reference is produced by sending the auxiliary outputs of the Mach-Zehnder interferometers through monochromators. The filtered pulses are thus stretched in time, and their interference serves as the lock-in reference. In this way the mechanical fluctuations of the setup are sensed and evaluated at the optical frequency set by the monochromator, and thus partially compensated for by the lock-in detection. The drawback of this method is that inter-pulse delays are limited by the monochromator resolution and throughput to about 10ps. In order to access the longer coherence and population times of semiconductor nanostructures, the use of an auxiliary $c w$ laser running through the interferometers and serving as a reference has been demonstrated [71]. In this latter contribution, the population signal of a semiconductor QW was measured in the form of a photocurrent. Photocurrent detection is particularly adapted for the study of optoelectronic devices such as photocells, since the obtained multidimensional spectra are directly related to the quantum yield of the device, providing insight into the photo-conversion mechanism [76]. Isolation of the FWM signal in the frequency domain by lock-in amplification provides additional advantages: intrinsic phase resolution, without the need of additional post-processing or measurements, and the possibility of simultaneously recording rephasing and nonrephasing signals on separated lock-in channels.

\subsection{Undersampling}

A general issue of multidimensional Fourier transform spectroscopy is the long duration of data acquisition. For a given spectral dimension, the resolution is given by $\frac{1}{\Delta t}$, where $\Delta t$ is the total inter-pulse delay that is probed. The spectral bandwidth is given by $\frac{1}{\delta t}$, where $\delta t$ is the delay step size. The number of time steps $N$ that needs to be taken on this axis is thus $N=\frac{\Delta t}{\delta t}$. When a regular spectrometer is used to obtain the emission frequency axis, the acquisition time scales as $N^{d-1}$, where $d=2$ or 3 for $2 \mathrm{D}$ or $3 \mathrm{D}$ spectra respectively. The situation is worse when all spectral axes are obtained through Fourier transform, as the acquisition time scales as $N^{d}$. To drastically reduce the total acquisition time, undersampling can be used to decrease the number of delay steps $N$. 
Undersampling makes use of larger delay steps (thus reducing the bandwidth) and an a priori knowledge of the signal frequency range (e.g., no coherence is expected to be induced outside of some known spectral region) to shift the centre of the acquisition bandwidth to larger frequencies. This strategy corresponds to setting the experiment in a rotating frame, centred on the shifted frequency. For a setup where time delays are controlled using mechanical translation stages, this is easily achieved by taking larger delay steps. To achieve undersampling using pulse-shaping methods, the frequency of the rotating frame is set by a proper phasing of the excitation pulses [35]. In experiments where a frequency selection of the signal is achieved using lock-in amplification (Section 3.2.3), a "physical" undersampling is achieved [75]. The signal evolves, as a function of time delays, in a rotating frame centred at the lock-in reference frequency. Indeed, in this case the demodulated signal evolves at the difference frequency between the optical frequency of the physical signal and of the reference (given by the monochromator wavelength in Refs $[75,74]$ or by the $c w$ reference laser in Ref [71]). Let us underline, though, that all these undersampling methods do not increase the bandwidth of the experiment: for a given delay step size, the bandwidth is always the same, only the centre of the spectral range is shifted.

Another strategy to reduce the number of sampling points is compressed sensing, based on the random sampling of a sparse data [77]. Implemented in the context of 2D coherent spectroscopy [78], this method enabled a reduction of the number of sampling points by a factor of 20 .

\section{Insight into the physics of semiconductor nanostructures}

In this section, various examples are given to illustrate the ability of MDCS to reveal some of the fundamental properties of semiconductor nanostructures. I discuss examples involving single and double quantum wells (QWs), large and small ensembles of quantum dots (QDs), semiconductor microcavities, and single- and multi-layered semiconductors.

\subsection{Heavy and light hole excitons in GaAs QWs}

The well-known, yet rich system of heavy and light hole excitons in GaAs QWs provides an ideal platform to demonstrate the ability of MDCS to assess coherences, coupling and many-body interactions in semiconductor materials. The excitonic coherence time, associated with its homogeneous linewidth, can be straightforwardly measured in a rephasing $2 \mathrm{D}$ spectrum $[7,79]$. An interesting example is given with the measurement of the polarization anisotropy of the homogeneous linewidth of excitons confined in [110]-oriented GaAs QWs [47].

In rephasing and non-rephasing $2 \mathrm{D}$ spectra, the various quantum pathways contributing to the nonlinear signal can be associated with different spectral features [80]. In particular, cross peaks demonstrate the presence of coupling between light hole and heavy hole excitons, as can be seen in Fig. 11 (a). Further measurements of zero- 
quantum spectra revealed the coherent nature of the heavy hole / light hole excitonic coupling, in the form of a Raman coherence (as described in Section 2.4) between the two species [12]. When the separation of real and imaginary parts is achieved, the line shape of the rephasing and non-rephasing 2D peaks enables identification of the contribution of many-body effects (Coulomb interaction between fermionic constituents of two excitons), namely Excitation Induced Shift (EIS) and Excitation Induced Dephasing (EID), to the nonlinear response of the QW [81]. Remarkably, Turner et al showed that many-body effects, attributed to the presence of a long-lived exciton population, can persist up to 40ps [82].

An asset of MDCS is its ability to measure interaction energy in a four-level system as shown in Fig. 5 (d). This ability is very useful to characterise biexcitonic resonance in semiconductor nano-structures. Due to polarisation selection rules, biexcitons are revealed when linear or cross-circularly polarised pulse sequences are used, whereas they do not appear with co-circular polarisation, as demonstrated in the case of GaAs QWs [83]. In Fig. 11, rephasing 2D spectra are shown for (a) a co-circular and (b) a cross-circular polarisation sequence. The heavy hole biexciton peak can be seen in the cross-circular case, separated in emission energy by the biexciton binding energy.

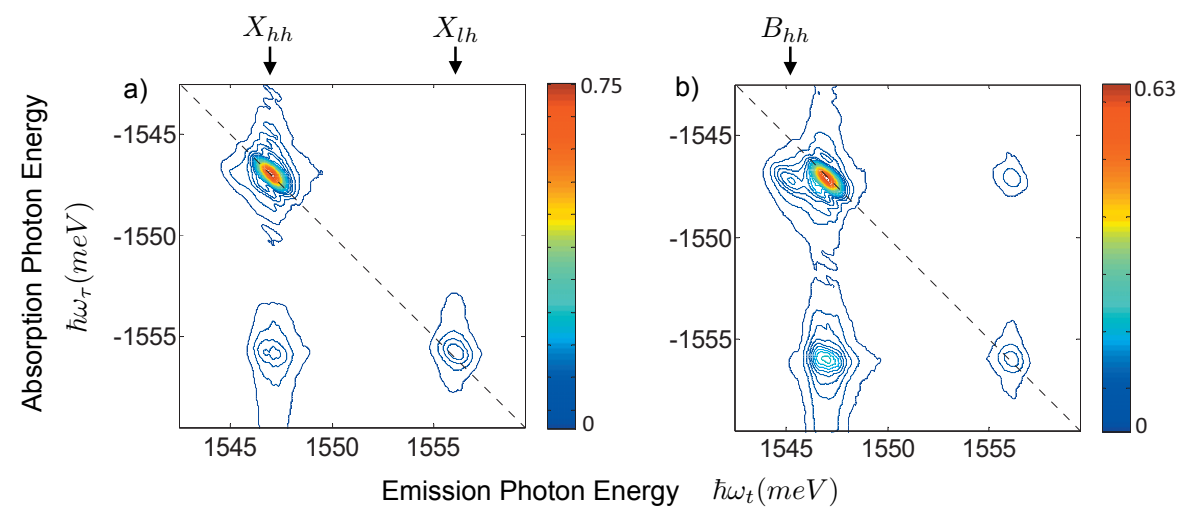

Figure 11. Absolute value of $2 \mathrm{D}$ rephasing spectra, recorded for (a) co-circular $\left(\sigma^{+}, \sigma^{+}, \sigma^{+}, \sigma^{+}\right)$, and (b) cross-circular $\left(\sigma^{-}, \sigma^{-}, \sigma^{+}, \sigma^{+}\right)$polarization sequences, in GaAs QWs at a temperature of approximately $7 \mathrm{~K}$. The delay between second and third pulses is $T=200 \mathrm{fs}$. A cross-peak shows that heavy hole (hh) and light hole (lh) excitons are coupled. A heavy hole biexciton peak is visible with the cross-circular polarization sequence. Adapted with permission from Ref. [83], copyrighted by the American Physical Society.

Two-photon coherences were observed in GaAs QWs using a phase-conjugated FWM experiment in 1996, and attributed to biexciton resonances [13]. Later on, Yang and Mukamel predicted that 2D spectra of such two-quantum signal would provide a sensitive probe of two-exciton interaction energy [14]. The first two-quantum spectrum of a GaAs QW was obtained by Stone et al [15], using a COLBERT-like apparatus (see Section 3.1.1) in a box geometry. Recorded using co-circular and cross-circular $S_{I I I}$ pulse sequences (the conjugated pulse arriving third on the sample), the two-quantum $2 \mathrm{D}$ 
spectra contained peaks associated with biexciton coherences, whose dephasing could be measured as a function of excitation density. This work demonstrated the ability of twoquantum MDCS to reveal many-body interactions in a semiconductor nanostructure. In further work, the signature of unbound two-exciton states was distinguished from the biexciton resonance, and many-body two-quantum coupling between the light hole and heavy hole excitons was revealed [84].

Turner et al recorded 3D spectra of the $S_{I}$ and $S_{I I I}$ signals, to ultimately isolate contributions from the different quantum pathways in the GaAs QW system [17]. The $S_{I} 3 \mathrm{D}$ spectrum and projections onto the three spectral axes provided straightforward view of the coherent coupling between light hole and heavy hole excitons, whereas the $S_{I I I} 3 \mathrm{D}$ spectra enabled the visualisation of biexciton pathways and a measure of the biexciton binding energy.

\subsection{Double QWs}

Epitaxial growth of semiconductor QWs provides a way to realise the "classroom" example of the double quantum well (DQW) geometry. QW width, depth and barrier size can be controlled with layer thicknesses and alloy content. The opportunity to experimentally study the coupling between two neighbouring QWs motivated several experiments, starting with the most common alloy: coupled GaAs QWs separated by AlGaAs barriers. Coupling between excitons in an asymmetric GaAs DQW was demonstrated using 2D [11] and 3D [18, 19] rephasing spectroscopy. However, the intermixing of heavy and light hole exciton coupling with the inter-well coupling resulted in complicated spectra that are difficult to interpret. Combining 3D spectroscopy with pathway-selective excitation provided a better isolation of the different contributions [42]. In Fig. 12 (a), a 3D rephasing spectrum is shown under broadband excitation conditions, for an asymmetric GaAs DQW with a $6 \mathrm{~nm}$ thick $\mathrm{Al}_{35} \mathrm{Ga}_{65}$ As barrier. Offdiagonal peaks, signatures of coupling between heavy and light holes between the two QWs are visible. Contribution to the coupling in the form of population decays, located in the plane $\hbar \omega_{2}$ (corresponding to $\hbar \omega_{T}$ in our previously introduced notation), dominate the signal. As a consequence, the observation of coherent coupling terms is hindered (only two peaks shown by a blue iso-surface, corresponding to coupling with the narrow well heavy hole exciton, are visible). Using a sequence of specifically spectrally-shaped pulses enables suppression of the population signal and selective excitation of the interwell coherent coupling pathways. As a result, four cross-peaks revealing coherent coupling between heavy and light hole excitons of each QW can be observed (Fig. 12 (b)).

Motivated by the possibility of a simpler system, closer to the "textbook" DQW, experiments using strained InGaAs asymmetric DQWs were performed [85]. Due to strain light holes are not confined in InGaAs QWs. Figure 13 (a) shows the 2D rephasing spectrum of coupled $\mathrm{In}_{0.05} \mathrm{Ga}_{0.95} \mathrm{As}$ QWs separated by a $10 \mathrm{~nm}$ thick GaAs barrier, with the second inter-pulse delay $T=200 \mathrm{fs}$, at approximately $10 \mathrm{~K}$. A cross- 

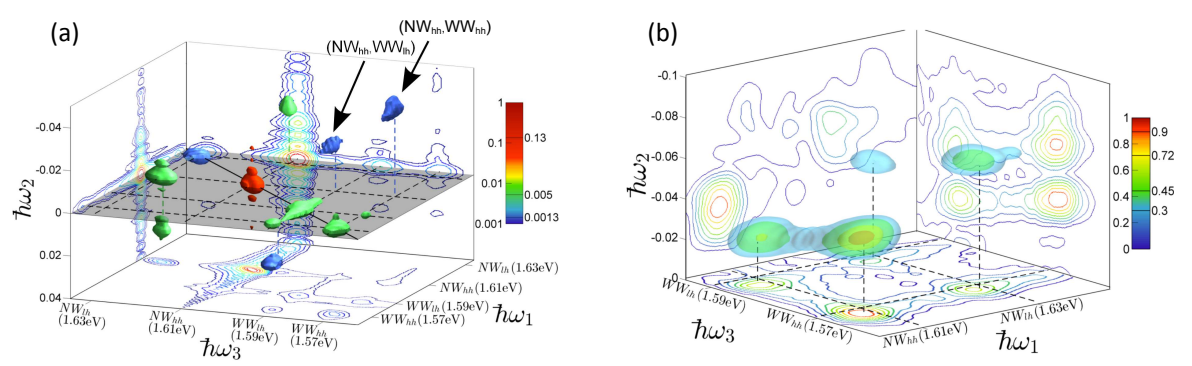

Figure 12. Rephasing 2D spectra (absolute value) of a GaAs asymmetric double QW, using (a) a broadband excitation spectrum for all pulses, and (b) individually spectrally-shaped pulses for pathway-selective excitation. $N W(W W)_{h h(l h)}$ stand for Narrow Well (Wide Well)-heavy (light) hole exciton. In (a), only two inter-well coherences are visible (blue iso-surfaces), as well as population terms (in the plane $\left.\hbar \omega_{2}=0\right)$. In (b), pathway-selective excitation suppresses population terms. The four coherent coupling peaks between the light hole and heavy hole excitons of each QW reveal the coherent nature of the inter-well coupling. Adapted from Ref. [42]. In this figure, axes $\left(\hbar \omega_{1}, \hbar \omega_{2}, \hbar \omega_{3}\right)$ correspond to axes $\left(-\hbar \omega_{\tau}, \hbar \omega_{T}, \hbar \omega_{t}\right)$ of our previously introduced notation.

peak (CP) demonstrates the existence of coupling between the narrow well and wide well excitons. When the real part of the data is plotted (Fig. 13 (b)), the cross peak exhibits a clear dispersive line shape. This information, combined with the existence of a coupling peak (2QCP) in the two-quantum spectrum (Fig. $13(\mathrm{~d})$ ), indicates that the coupling between the two QW excitons is of a coherent type, described by a diamondlevel system as depicted in Fig 5 (d). In this case, the interaction energy is attributed to inter-well many-body excitonic interactions [85]. In this situation, one would expect non-zero-frequency peaks to show up in the zero-quantum spectrum of Fig. 5 (c). The absence of such coupling peaks was attributed to population terms dominating the signal. This interpretation was supported by measurements of $2 \mathrm{D}$ rephasing spectra for various values of the delay $T$ [85], revealing the increasingly dominating contribution of population relaxation from the narrow to the wide well exciton as $T$ is increased to a few picoseconds. Pathway-selective excitation [42] would be necessary to suppress population terms and reveal non-zero $\hbar \omega_{T}$ peaks in a zero-quantum or $3 \mathrm{D}$ spectrum. Simulations based on OBEs for a diamond system added with two-exciton states for each QW were able to reproduce all experimental features, as shown in Fig. 13 (e)-(h). It should be noted that InGaAs/GaAs QWs are shallower than GaAs/AlGaAs QWs, reason for which coupling is observed for a barrier as thick as $10 \mathrm{~nm}$. Very different 2D spectra were observed for InGaAs DQW with a thinner barrier of $5 \mathrm{~nm}$ [86], suggesting a strong modification of the excitonic structure. While these latter results are not understood yet, they imply that care should be taken when designing devices with closely packed stacks of shallow InGaAs QWs, such as semiconductor microcavities. 


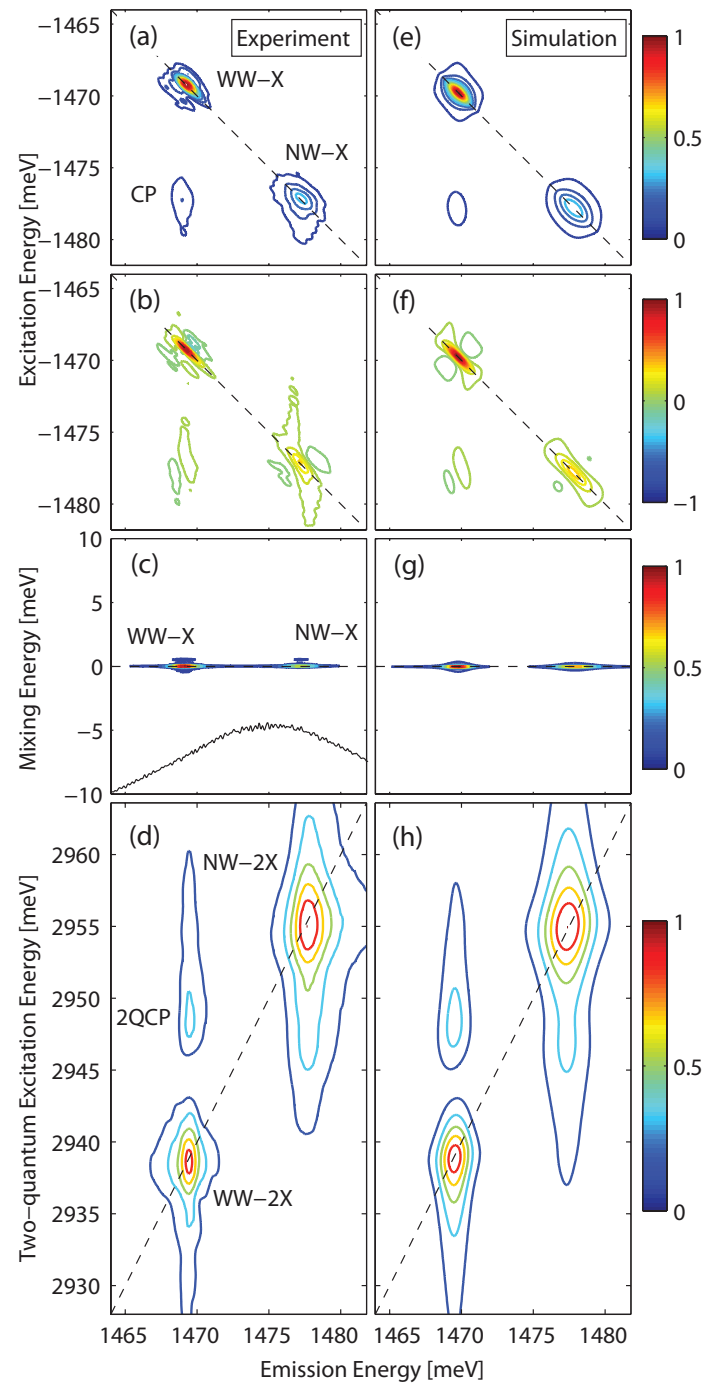

Figure 13. 2D spectra measured on an InGaAs asymmetric double QW, at a temperature of approximately 10K. (a) Absolute value of the 2D rephasing spectrum, at $T=200 \mathrm{fs}$, showing a coupling peak (CP) between the wide well exciton (WW$\mathrm{X})$ and the narrow well exciton (NW-X). (b) Real part of the same data, revealing the dispersive line shape of the CP. (c) Zero-quantum spectrum (absolute value), dominated by population terms at $\hbar \omega_{T}=0$. The solid line shows the excitation spectrum. (c) Two-quantum 2D spectrum (absolute value), at $\tau=200 \mathrm{fs}$, revealing an inter-well two-quantum coherence (2QCP). (e)-(h) 2D spectra calculated from OBEs, using a single set of parameters for all spectra. Adapted from Ref. [85], copyrighted by the American Physical Society.

\subsection{Large $Q D$ ensembles}

Confined in three dimensions of space, QD excitons are seen by many as candidates to serve as quantum information bits (qubits). While a lot of attention has been devoted to the coherent optical manipulation of excitons in single or double QD systems (for a review, see Ref. [87]), the ability to coherently address larger ensembles is the key towards scalability of QD-exciton-based quantum algorithms. However, large 


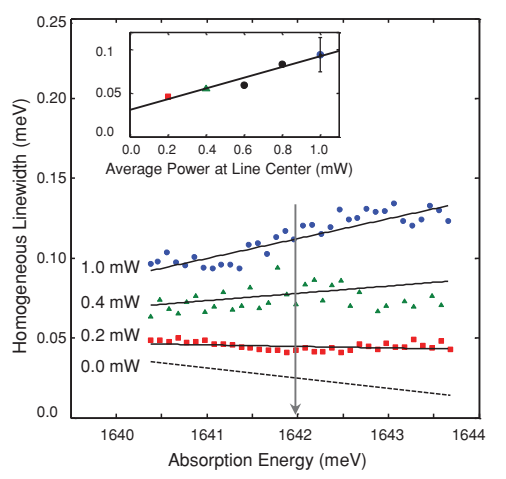

Figure 14. Homogeneous linewidth as a function of excitation density and absorption energy, extracted from 2D rephasing spectra across the inhomogeneous distribution, for interfacial GaAs QDs at a temperature of $6 \mathrm{~K}$. The gray vertical arrow indicates the line centre of the inhomogeneous distribution. Inset: homogeneous linewidth, at line centre, as a function of excitation power. Extrapolation provides the zero excitation density homogeneous linewidth, corresponding to a QD radiative lifetime of $68 \mathrm{ps}$. Adapted from Ref. [48], copyrighted by the American Physical Society.

ensembles of semiconductor QDs provide an archetypical example of inhomogeneously broadened system, for the inhomogeneous size distribution translates into a distribution of transition energies. The pulse sequence of a MDCS experiment can be seen as initialisation / readout sequence of the QD exciton coherence. By probing the coherence time, MDCS enables the measurement of the homogeneous linewidth despite the inhomogeneous distribution, as introduced in Section 2.2. As an example, Moody et al have recorded 2D rephasing spectra to measure the homogeneous linewidth of interfacial GaAs QD excitons, as a function of temperature and excitation density [48]. These two measurements enabled quantification of exciton-phonon, respectively excitonexciton interaction energies, and provided insight into the underlying mechanisms. As an example, Fig. 14 shows the homogeneous linewidth as a function of excitation density. The linewidth is also plotted as a function of absorption energy (related to the QD size), since this information is readily available in a 2D rephasing spectrum. This figure shows that excitation-induced dephasing (EID) is more pronounced in higher energy (smaller size) QDs, suggesting that intra-QD exciton interactions is the dominant EID mechanism. Still using 2D rephasing spectra but with cross-polarized pulse sequences, biexciton coherences were studied in several kind of epitaxial QDs (self-assembled InAs QDs annealed at different temperatures, and interfacial GaAs QDs) [88]. As can be seen in Fig. 15 (a) with the example of InAs QDs, a 2D rephasing spectrum enables the clear separation of the biexciton peak, despite the inhomogeneous distribution of QDs. Moreover, the biexciton binding energy can be precisely measured, and this as a function of emission energy (Fig 15 (b)-(c)), exhibiting very different dependencies for self-assembled InAs and interfacial GaAs QDs. These results show that the biexciton binding energy can be tuned using annealing temperature in InAs QDs and using QD size in GaAs QD, which is of importance for the fabrication of QD samples with deterministic 
biexciton binding energies. Other examples of MDCS experiments on epitaxial QDs include: the study of coupling dynamics between GaAs QW and interfacial QDs [89] ; the use of zero-quantum (Raman) spectra to determine the role of geometrical effects on the fine structure splitting and dephasing in InAs QDs [90]. MDCS has also been used to study colloidal QDs (referred to as nanocrystals in the chemistry community). In CdSe nanocrystals, the large exciton binding energy has enabled MDCS experiments to characterise exciton dephasing and dynamics up to room temperature [91, 92, 93]. Interestingly, a 2D coherent photocurrent spectroscopy experiment has been used to study a PbS colloidal QD photocell [76]. In this work, non-instantaneous EIS was observed only when the excitation energy was above three times the band gap, which was ascribed to a multiple exciton generation (MEG) mechanism. More generally, this latter work showed the potential of 2D coherent photocurrent spectroscopy to study the photo-conversion mechanisms that affect the functioning of optoelectronic devices.
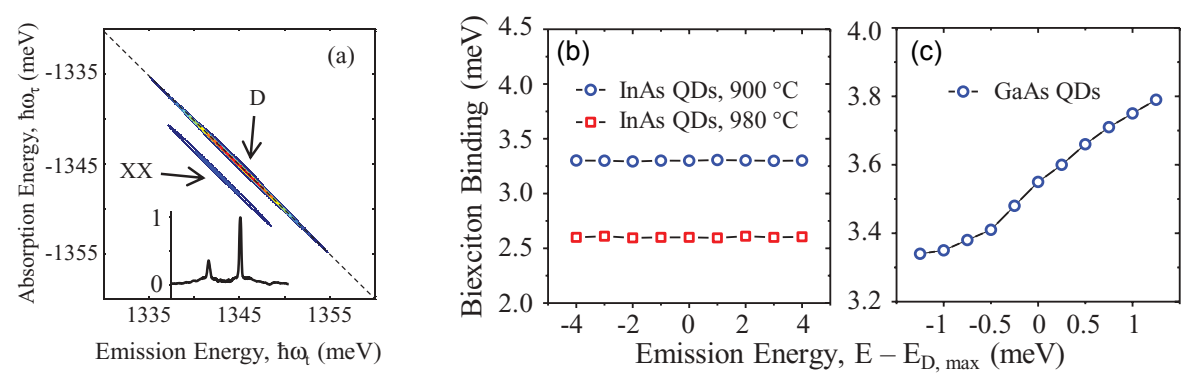

Figure 15. Rephasing 2D spectrum (absolute value) of self-assembled InAs QDs annealed at $900^{\circ} \mathrm{C}$, recorded at a temperature of $10 \mathrm{~K}$, with a cross-linear polarization sequence. The spectral features are a diagonal peak (D) and a biexciton peak (XX). An horizontal slice shown as inset, exemplifies how the biexciton binding energy can be precisely measured despite the inhomogeneous QD distribution. Biexciton binding energies are plotted as a function of emission energy for InAs QDs annealed at two different temperatures (c) and GaAs interfacial QDs. Adapted with permission from Ref. [88], copyrighted by the American Physical Society.

\subsection{Single or few $Q D s$}

Taking advantage of the ability of Heterodyne Spectral Interferometry (HSI) [63] to isolate nonlinear signals from low-dimensional nanostructures (see Section 3.2.1), Langbein and co-workers have performed pioneering 2D coherent spectroscopy experiments on single and few QDs. Studying excitons confined in monolayer fluctuations of GaAS QWs and in self assembled CdTe QDs [94], these authors have observed and measured fine structure splitting and coherent coupling between different excitonic states of a single QD. Even more interestingly, by spatially resolving the FWM spectra, the same group identified coherent coupling between excitons confined in separated GaAs monolayer islands [65]. The corresponding 2D spectrum is shown in Fig. 16, where cross-peaks between individual transitions are clearly visible. By measuring 
the relative coupling strength as a function of inter-dot distance, coherent coupling was observed up to distances of $1 \mu \mathrm{m}$. Relying on the phase structure of the crosspeaks, the coherent coupling mechanism was attributed to a two-exciton interaction, as depicted by the four-level diagram of Fig. 5 (d) - similarly to the coherent excitonic coupling observed between QWs for short $T$ discussed in Section 4.2. Further work with polarization resolution enabled the determination of polarization selection rules for quantum pathways involving biexcitons and fine-structure-splitted states in single QDs [95]. A similar experiment realised on InGaAs QDs embedded in a micro-pillar cavity demonstrated the cavity-mediated coherent coupling of up to three excitons confined in separated QDs [68]. If QD excitonic transitions are used as qubits, the coherent optical control and readout of a small ensemble QDs demonstrated in these contributions can be seen as an interesting step toward the implementation of quantum algorithms.

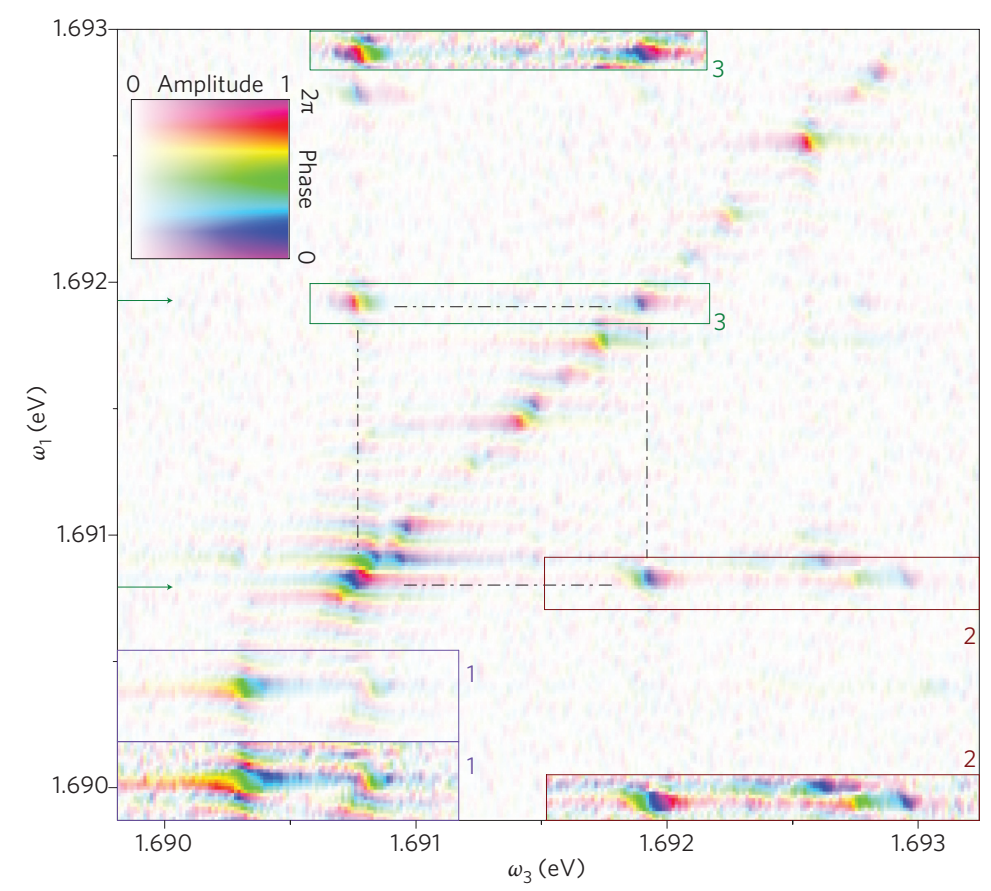

Figure 16. 2D rephasing spectrum of excitons confined in monolayer fluctuations of a 5nm-thick GaAs QW. Amplitude (saturation) and phase (hue) of the FWM signal are displayed. Insets 1-3 show regions 1-3 with a four times smaller amplitude scale. The dashed-dotted square shows coupled excitons. Hyperspectral imaging reveals that these excitons are confined in separated monolayer islands. Analysis of the phaseresolved line shape reveals the coherent nature of the inter-QD coupling, which can be modelled via a two-exciton interaction energy in a four-level 'diamond' system. Adapted by permission from Macmillan Publishers Ltd: Nature Photonics (Ref. [65]), copyright $(2011)$. In this figure, $\left(\omega_{1}, \omega_{3}\right)$ corresponds to $\left(-\omega_{\tau}, \omega_{t}\right)$ of our previously introduced notation. 


\subsection{Semiconductor microcavities}

When semiconductor QWs are embedded in a high-quality semiconductor microcavity, strong light-matter coupling gives rise to new eigenmodes, the exciton-polaritons [96]. An abundant literature explores the very rich physics of this optoelectronic system and its potential applications. Yet, the topic of polariton-polariton interactions and the role of biexciton resonance therein - remains a current topic of research and debate [97, 98, 99] into which MDCS promises to bring insight. First phaseresolved FWM experiments were realised in the perspective of observing the polaritonic ghost branch $[66,67]$, detecting signal in the $2 k_{2}-k_{1}$ direction, in combination with heterodyne spectral interferometry. Using intrinsically phase-stable, pulse-shapingbased 2D spectroscopy [35], Wen et al were able to obtain two-quantum, three-quantum and four-quantum 2D spectra of polaritons, revealing polariton-polariton correlations up to the fourth order [59] - a higher order than previously observed in a simple QW system [100]. This work showed that MDCS can see signatures of the biexciton resonance in polariton-polariton interactions, a feature confirmed by the observation of a polaritonic Feshbach resonance [99]. Recent experimental work carried out using using the box geometry [101] mapped the polaritonic anti-crossing with rephasing and nonrephasing 2D spectra, also observing biexcitonic features. Very recently, Takemura et al recorded one-quantum and two-quantum spectra to reveal the qualitative contribution of polaritonic interactions (self and cross interactions between upper and lower polaritons) to the nonlinear optical response [69]. These experiments show that MDCS has a strong potential for the study of many-body interactions in semiconductor microcavities. In particular, a line shape analysis of the real part of $2 \mathrm{D}$ spectra would provide a direct, sign-resolved measurement of polariton-polariton interactions. Let us also note that the MDCS experiments with exciton-polaritons were so far carried out in non-collinear geometries, which mixes different polariton modes and restrict the excitation angles to those satisfying energy and momentum conservation rules imposed by the polaritonic dispersion curve. Collinear experiments (Section 3.2) could potentially enable FWM of polaritons with identical momentum and arbitrary excitation angle.

\subsection{Layered semiconductors}

Transition metal dichalcogenides (TMDs) are currently attracting a tremendous attention due to fascinating properties - opening of a direct bandgap [102], spin and valley effects [103, 104], large exciton and biexciton binding energies [105, 106] when produced in atomically-thin layers. While the spectral response of monolayer TMDs was assumed to be dominated by inhomogeneous broadening, the value of the intrinsic homogeneous linewidth - and thus of the dephasing time - of the excitonic transition had not been measured until recently. Using MDCS, Moody et al have measured the homogeneous linewidth of monolayer $W S e_{2}$ as a function of excitation density and temperature [107]. The authors extracted a zero-density, zero-temperature homogeneous linewidth of $1.6 \mathrm{meV}$, nearly two orders of magnitude smaller than the 
inhomogeneous linewidth measured with photoluminescence spectroscopy. Knowing this value is essential for the design of future TMD-based devices [108], as it sets the time scale during which excitons can be coherently manipulated.

Also of interest are excitons in coupled multi-layer materials [109]. Biexciton binding energy, homogeneous linewidth, and coupling between excitonic species in layered GaSe [110], and exciton dephasing in layered InSe [111], have been recently measured using MDCS by Dey et al.

\section{Conclusion}

The author hopes that the material presented in this review has convinced the reader that MDCS is a powerful tool to reveal the fundamental properties of semiconductor nanostructures. In particular, it was shown how MDCS can provide a direct access to the homogeneous linewidth (or dephasing time) of excitonic transitions, can assess and distinguish coherent and incoherent coupling mechanisms, and highlight the role of many-body interactions in the nonlinear optical response of the nanostructures. Examples were given for a broad range of nanostructures : quantum wells, quantum dots, exciton-polaritons, and layered semiconductors. Different experimental approaches were detailed, adapted to several situations, with the detected signal in the form of a radiated optical field or a photocurrent.

I foresee a bright future for MDCS with materials and structures that have been barely studied with the technique so far. In particular, I am thinking about: TMDs [107], whose fundamental properties such as intrinsic homogeneous linewidth can be measured with MDCS ; semiconductor microcavities [59, 101, 69], where MDCS can help bringing insight into the rich physics of polariton-polariton interactions ; small ensembles of QDs [95, 68], within which the control of coherent coupling mechanisms may open the way to scalable quantum information processors [2]. Furthermore, the recent development of photocurrent-based MDCS [71, 76] promises to shed a new light on the nonlinear optical response of optoelectronic devices in operating conditions.

While a large part of the experiments discussed in this paper involve the use of Titanium Sapphire oscillators as pulsed laser sources (well adapted to the near infrared range of GaAs based semiconductors), efforts are pursued towards experiments with broader band excitation, shorter wavelengths, and mixed wavelength [112]. For example, reaching the UV range opens the perspective of MDCS of GaN based semiconductors.

The use of entangled photons in MDCS has also been theoretically discussed, and promises to be a sensitive and selective way to probe coherences between transitions, beyond what is possible with classical light $[113,114,115,116]$.

Another theoretical proposal discusses the generation of $2 \mathrm{D}$ spectra using a frequency-filtered version of the Hanbury Brown-Twiss interferometer [117, 118]. The resulting two-photon $2 \mathrm{D}$ spectra are predicted to be specifically adapted to the characterisation of quantum systems such as QDs in microcavities. 


\section{Acknowledgement}

The author would like to thank S.T. Cundiff and R. Singh for proofreading the manuscript. The author acknowledges support by the Swiss National Science Foundation (SNSF).

\section{References}

[1] Jerome Faist, Federico Capasso, Deborah L. Sivco, Carlo Sirtori, Albert L. Hutchinson, and Alfred Y. Cho. Quantum Cascade Laser. Science, 264(5158):553-556, April 1994.

[2] Eliana Biolatti, Rita C. Iotti, Paolo Zanardi, and Fausto Rossi. Quantum Information Processing with Semiconductor Macroatoms. Phys. Rev. Lett., 85(26):5647, 2000.

[3] Gaël Nardin, Travis M. Autry, Galan Moody, Rohan Singh, Hebin Li, and Steven T. Cundiff. Multi-dimensional coherent optical spectroscopy of semiconductor nanostructures: Collinear and non-collinear approaches. Journal of Applied Physics, 117(11):112804, March 2015.

[4] Shaul Mukamel. Principles of Nonlinear Optical Spectroscopy. Oxford University Press, April 1999.

[5] Robert W. Boyd. Nonlinear Optics. Academic Press, January 2003.

[6] N. A. Kurnit, I. D. Abella, and S. R. Hartmann. Observation of a Photon Echo. Phys. Rev. Lett., 13(19):567-568, November 1964.

[7] Mark E. Siemens, Galan Moody, Hebin Li, Alan D. Bristow, and Steven T. Cundiff. Resonance lineshapes in two-dimensional Fourier transform spectroscopy. Opt. Express, 18(17):17699$17708,2010$.

[8] M. D. Webb, S. T. Cundiff, and D. G. Steel. Observation of time-resolved picosecond stimulated photon echoes and free polarization decay in GaAs/AlGaAs multiple quantum wells. Phys. Rev. Lett., 66(7):934-937, February 1991.

[9] M. Koch, J. Feldmann, G. von Plessen, E. O. Göbel, P. Thomas, and K. Köhler. Quantum beats versus polarization interference: An experimental distinction. Phys. Rev. Lett., 69(25):36313634, 1992.

[10] K. Bott, O. Heller, D. Bennhardt, S. T. Cundiff, P. Thomas, E. J. Mayer, G. O. Smith, R. Eccleston, J. Kuhl, and K. Ploog. Influence of exciton-exciton interactions on the coherent optical response in GaAs quantum wells. Phys. Rev. B, 48(23):17418-17426, 1993.

[11] Xiaoqin Li, Tianhao Zhang, Shaul Mukamel, Richard P. Mirin, and Steven T. Cundiff. Investigation of electronic coupling in semiconductor double quantum wells using coherent optical two-dimensional Fourier transform spectroscopy. Solid State Comm., 149(9-10):361366, March 2009.

[12] Lijun Yang, Tianhao Zhang, Alan D. Bristow, Steven T. Cundiff, and Shaul Mukamel. Isolating excitonic Raman coherence in semiconductors using two-dimensional correlation spectroscopy. The Journal of Chemical Physics, 129(23):234711, December 2008.

[13] K. B. Ferrio and D. G. Steel. Observation of the ultrafast two-photon coherent biexciton oscillation in a GaAs/AlGaAs multiple quantum well. Phys. Rev. B, 54(8):R5231-R5234, 1996.

[14] Lijun Yang and Shaul Mukamel. Two-Dimensional Correlation Spectroscopy of Two-Exciton Resonances in Semiconductor Quantum Wells. Phys. Rev. Lett., 100(5):057402, 2008.

[15] Katherine W. Stone, Kenan Gundogdu, Daniel B. Turner, Xiaoqin Li, Steven T. Cundiff, and Keith A. Nelson. Two-Quantum 2d FT Electronic Spectroscopy of Biexcitons in GaAs Quantum Wells. Science, 324(5931):1169-1173, May 2009.

[16] Steven T. Cundiff. Optical three dimensional coherent spectroscopy. Phys. Chem. Chem. Phys., 16(18):8193-8200, April 2014.

[17] Daniel B. Turner, Katherine W. Stone, Kenan Gundogdu, and Keith A. Nelson. Three- 
dimensional electronic spectroscopy of excitons in GaAs quantum wells. The Journal of Chemical Physics, 131(14):144510, October 2009.

[18] J. A. Davis, C. R. Hall, L. V. Dao, K. A. Nugent, H. M. Quiney, H. H. Tan, and C. Jagadish. Three-dimensional electronic spectroscopy of excitons in asymmetric double quantum wells. J. Chem. Phys, 135(4):044510-044510-9, July 2011.

[19] C. R. Hall, J. O. Tollerud, H. M. Quiney, and J. A. Davis. Three-dimensional electronic spectroscopy of excitons in asymmetric double quantum wells. New J. Phys., 15(4):045028, April 2013.

[20] Hebin Li, Alan D. Bristow, Mark E. Siemens, Galan Moody, and Steven T. Cundiff. Unraveling quantum pathways using optical 3d Fourier-transform spectroscopy. Nat. Commun., 4:1390, January 2013.

[21] Richard Ernst, G Bodenhausen, and A Wokaun. Principles of nuclear magnetic resonance in one and two dimensions. Clarendon Press ;;Oxford University Press, Oxford [Oxfordshire] ;New York, 1987.

[22] Peter Hamm and Martin Zanni. Concepts and Methods of 2D Infrared Spectroscopy. Cambridge University Press, Cambridge, UK ; New York, 1 edition edition, March 2011.

[23] David M. Jonas. Two-Dimensional Femtosecond Spectroscopy. Annual Review of Physical Chemistry, 54(1):425-463, 2003.

[24] D. Grischkowsky, SÃ̈̈ren Keiding, Martin van Exter, and Ch. Fattinger. Far-infrared timedomain spectroscopy with terahertz beams of dielectrics and semiconductors. Journal of the Optical Society of America B, 7(10):2006, October 1990.

[25] P. Y. Han, M. Tani, M. Usami, S. Kono, R. Kersting, and X.-C. Zhang. A direct comparison between terahertz time-domain spectroscopy and far-infrared Fourier transform spectroscopy. Journal of Applied Physics, 89(4):2357-2359, February 2001.

[26] W. Kuehn, K. Reimann, M. Woerner, and T. Elsaesser. Phase-resolved two-dimensional spectroscopy based on collinear n-wave mixing in the ultrafast time domain. The Journal of Chemical Physics, 130(16):164503, April 2009.

[27] W. Kuehn, K. Reimann, M. Woerner, T. Elsaesser, and R. Hey. Two-Dimensional Terahertz Correlation Spectra of Electronic Excitations in Semiconductor Quantum Wells. J. Phys. Chem. B, 115(18):5448-5455, 2011.

[28] W. Kuehn, K. Reimann, M. Woerner, T. Elsaesser, R. Hey, and U. Schade. Strong Correlation of Electronic and Lattice Excitations in GaAs/AlGaAs Semiconductor Quantum Wells Revealed by Two-Dimensional Terahertz Spectroscopy. Phys. Rev. Lett., 107(6):067401, August 2011.

[29] T. K. Paraïso, D. Sarchi, G. Nardin, R. Cerna, Y. Leger, B. Pietka, M. Richard, O. El Daïf, F. Morier-Genoud, V. Savona, and B. Deveaud-Plédran. Enhancement of microcavity polariton relaxation under confinement. Phys. Rev. B, 79(4):045319, January 2009.

[30] Akshay Singh, Galan Moody, Sanfeng Wu, Yanwen Wu, Nirmal J. Ghimire, Jiaqiang Yan, David G. Mandrus, Xiaodong Xu, and Xiaoqin Li. Coherent Electronic Coupling in Atomically Thin MoSe2. Phys. Rev. Lett., 112(21):216804, May 2014.

[31] M. Khalil, N. Demirdöven, and A. Tokmakoff. Coherent 2d IR Spectroscopy: Molecular Structure and Dynamics in Solution. J. Phys. Chem. A, 107(27):5258-5279, 2003.

[32] Tobias Brixner, T. Mančal, Igor V. Stiopkin, and Graham R. Fleming. Phase-stabilized twodimensional electronic spectroscopy. J. Chem. Phys., 121(9):4221-4236, August 2004.

[33] Tianhao Zhang, Camelia Borca, Xiaoqin Li, and Steven Cundiff. Optical two-dimensional Fourier transform spectroscopy with active interferometric stabilization. Opt. Express, 13(19):7432$7441,2005$.

[34] A. D. Bristow, D. Karaiskaj, X. Dai, T. Zhang, C. Carlsson, K. R. Hagen, R. Jimenez, and S. T. Cundiff. A versatile ultrastable platform for optical multidimensional Fourier-transform spectroscopy. Rev. Sci. Instrum., 80(7):073108, July 2009.

[35] Daniel B. Turner, Katherine W. Stone, Kenan Gundogdu, and Keith A. Nelson. Invited Article: The coherent optical laser beam recombination technique (COLBERT) spectrometer: Coherent 
multidimensional spectroscopy made easier. Review of Scientific Instruments, 82(8):081301, August 2011.

[36] L. Lepetit, G. Chériaux, and M. Joffre. Linear techniques of phase measurement by femtosecond spectral interferometry for applications in spectroscopy. J. Opt. Soc. Am. B, 12(12):2467-2474, 1995.

[37] Hebin Li, Austin P. Spencer, Andrew Kortyna, Galan Moody, David M. Jonas, and Steven T. Cundiff. Pulse Propagation Effects in Optical 2d Fourier-Transform Spectroscopy: Experiment. J. Phys. Chem. A, 117(29):6279-6287, July 2013.

[38] Hebin Li, Galan Moody, and Steven T. Cundiff. Reflection optical two-dimensional Fouriertransform spectroscopy. Opt. Express, 21(2):1687-1692, January 2013.

[39] Alexandra Nemeth, Jaroslaw Sperling, Jürgen Hauer, Harald F. Kauffmann, and Franz Milota. Compact phase-stable design for single- and double-quantum two-dimensional electronic spectroscopy. Optics Letters, 34(21):3301, November 2009.

[40] Peifang Tian, Dorine Keusters, Yoshifumi Suzaki, and Warren S. Warren. Femtosecond PhaseCoherent Two-Dimensional Spectroscopy. Science, 300(5625):1553-1555, June 2003.

[41] Joshua C. Vaughan, Thomas Hornung, T. Feurer, and Keith A. Nelson. Diffraction-based femtosecond pulse shaping with a two-dimensionalspatial light modulator. Opt. Lett., 30(3):323-325, February 2005.

[42] Jonathan O Tollerud, Christopher R Hall, and Jeffrey A Davis. Isolating quantum coherence using coherent multi-dimensional spectroscopy with spectrally shaped pulses. Opt. Express, 22(6):6719-6733, March 2014.

[43] P. Borri, W. Langbein, S. Schneider, U. Woggon, R. L. Sellin, D. Ouyang, and D. Bimberg. Ultralong Dephasing Time in InGaAs Quantum Dots. Phys. Rev. Lett., 87(15):157401, 2001.

[44] D. Birkedal, K. Leosson, and J. M. Hvam. Long Lived Coherence in Self-Assembled Quantum Dots. Phys. Rev. Lett., 87(22):227401, November 2001.

[45] Victor Volkov, Roland Schanz, and Peter Hamm. Active phase stabilization in Fourier-transform two-dimensional infrared spectroscopy. Opt. Lett., 30(15):2010-2012, August 2005.

[46] P. Dey, J. Paul, J. Bylsma, S. Deminico, and D. Karaiskaj. Continuously tunable optical multidimensional Fourier-transform spectrometer. Review of Scientific Instruments, 84(2):023107, February 2013.

[47] Rohan Singh, Travis M. Autry, Gaël Nardin, Galan Moody, Hebin Li, Klaus Pierz, Mark Bieler, and Steven T. Cundiff. Anisotropic homogeneous linewidth of the heavy-hole exciton in (110)oriented GaAs quantum wells. Phys. Rev. B, 88(4):045304, 2013.

[48] G. Moody, M. E. Siemens, A. D. Bristow, X. Dai, D. Karaiskaj, A. S. Bracker, D. Gammon, and S. T. Cundiff. Exciton-exciton and exciton-phonon interactions in an interfacial GaAs quantum dot ensemble. Phys. Rev. B, 83(11):115324, March 2011.

[49] G. Moody, R. Singh, H. Li, I. A. Akimov, M. Bayer, D. Reuter, A. D. Wieck, and S. T. Cundiff. Fifth-order nonlinear optical response of excitonic states in an InAs quantum dot ensemble measured with two-dimensional spectroscopy. Phys. Rev. B, 87(4):045313, January 2013.

[50] M. Khalil, N. Demirdöven, and A. Tokmakoff. Obtaining Absorptive Line Shapes in TwoDimensional Infrared Vibrational Correlation Spectra. Phys. Rev. Lett., 90(4):047401, January 2003.

[51] Sarah M. Gallagher Faeder and David M. Jonas. Two-Dimensional Electronic Correlation and Relaxation Spectra: Theory and Model Calculations. J. Phys. Chem. A, 103(49):10489-10505, 1999.

[52] Alan D. Bristow, Denis Karaiskaj, Xingcan Dai, and Steven T. Cundiff. All-optical retrieval of the global phase for two-dimensionalFourier-transform spectroscopy. Opt. Express, 16(22):1801718027, October 2008.

[53] Ellen H. G. Backus, Sean Garrett-Roe, and Peter Hamm. Phasing problem of heterodynedetected two-dimensional infrared spectroscopy. Opt. Lett., 33(22):2665-2667, November 2008.

[54] Sang-Hee Shim and Martin T. Zanni. How to turn your pump-probe instrument into a 
multidimensional spectrometer: 2d IR and Vis spectroscopies via pulse shaping. Phys. Chem. Chem. Phys., 11(5):748-761, February 2009.

[55] Erik M. Grumstrup, Sang-Hee Shim, Matthew A. Montgomery, Niels H. Damrauer, and Martin T. Zanni. Facile collection of two-dimensional electronic spectra using femtosecond pulse-shaping Technology. Opt. Express, 15(25):16681-16689, 2007.

[56] Sang-Hee Shim, David B. Strasfeld, Eric C. Fulmer, and Martin T. Zanni. Femtosecond pulse shaping directly in the mid-IR using acousto-optic modulation. Opt. Lett., 31(6):838-840, March 2006.

[57] Jacob M. Nite, Jenée D. Cyran, and Amber T. Krummel. Active Bragg angle compensation for shaping ultrafast mid-infrared pulses. Opt. Express, 20(21):23912-23920, October 2012.

[58] Daniele Brida, Cristian Manzoni, and Giulio Cerullo. Phase-locked pulses for two-dimensional spectroscopy by a birefringent delay line. Opt. Lett., 37(15):3027-3029, August 2012.

[59] P. Wen, G. Christmann, J. J. Baumberg, and Keith A. Nelson. Influence of multi-exciton correlations on nonlinear polariton dynamics in semiconductor microcavities. New J. Phys., 15(2):025005, February 2013.

[60] Jeffrey A. Myers, Kristin L. Lewis, Patrick F. Tekavec, and Jennifer P. Ogilvie. Two-color two-dimensional Fourier transform electronic spectroscopy with a pulse-shaper. Opt. Express, 16(22):17420-17428, October 2008.

[61] Verena Kohnle. Nonlinear Spectroscopy of a Dissipative Polariton Quantum Fluid. PhD thesis, EPFL, 2011.

[62] Antonio Mecozzi and Jesper Mork. Transient and time-resolved four-wave mixing with collinear pump and probe pulses using the heterodyne technique. Pure Appl. Opt., 7(2):335, March 1998.

[63] Wolfgang Langbein and Brian Patton. Heterodyne spectral interferometry for multidimensional nonlinear spectroscopy of individual quantum systems. Opt. Lett., 31(8):1151-1153, 2006.

[64] Wolfgang Langbein and Brian Patton. Transient coherent nonlinear spectroscopy of single quantum dots. J. Phys.: Condens. Matter, 19(29):295203, July 2007.

[65] J. Kasprzak, Patton B., Savona V., and Langbein W. Coherent coupling between distant excitons revealed by two-dimensional nonlinear hyperspectral imaging. Nature Photon., 5(1):57-63, January 2011.

[66] V. Kohnle, Y. Léger, M. Wouters, M. Richard, M. T. Portella-Oberli, and B. Deveaud-Plédran. From Single Particle to Superfluid Excitations in a Dissipative Polariton Gas. Phys. Rev. Lett., 106(25):255302, June 2011.

[67] V. Kohnle, Y. Léger, M. Wouters, M. Richard, M. T. Portella-Oberli, and B. Deveaud. Four-wave mixing excitations in a dissipative polariton quantum fluid. Phys. Rev. B, 86(6):064508, 2012.

[68] F. Albert, K. Sivalertporn, J. Kasprzak, M. Strauss§, C. Schneider, S. Höfling, M. Kamp, A. Forchel, S. Reitzenstein, E. A. Muljarov, and W. Langbein. Microcavity controlled coupling of excitonic qubits. Nat. Commun., 4:1747, 2013.

[69] N. Takemura, S. Trebaol, M. D. Anderson, V. Kohnle, Y. Léger, D. Y. Oberli, M. T. PortellaOberli, and B. Deveaud. 2d Fourier Transform Spectroscopy of exciton-polaritons and their interactions. arXiv:1506.01964 [cond-mat], June 2015. arXiv: 1506.01964.

[70] J. A. Davis, L. V. Dao, M. T. Do, P. Hannaford, K. A. Nugent, and H. M. Quiney. Noninterferometric Two-Dimensional Fourier-Transform Spectroscopy of Multilevel Systems. Phys. Rev. Lett., 100(22):227401, June 2008.

[71] Gaël Nardin, Travis M. Autry, Kevin L. Silverman, and S. T. Cundiff. Multidimensional coherent photocurrent spectroscopy of a semiconductor nanostructure. Opt. Express, 21(23):2861728627, November 2013.

[72] Dorine Keusters, Howe-Siang Tan, and Warren, W.S. Role of Pulse Phase and Direction in Two-Dimensional Optical Spectroscopy. J. Phys. Chem. A, 103(49):10369-10380, 1999.

[73] Martin Aeschlimann, Tobias Brixner, Alexander Fischer, Christian Kramer, Pascal Melchior, Walter Pfeiffer, Christian Schneider, Christian Strüber, Philip Tuchscherer, and Dmitri V. 
Voronine. Coherent Two-Dimensional Nanoscopy. Science, 333(6050):1723-1726, September 2011.

[74] Patrick F. Tekavec, Geoffrey A. Lott, and Andrew H. Marcus. Fluorescence-detected twodimensional electronic coherence spectroscopy by acousto-optic phase modulation. The Journal of Chemical Physics, 127(21):214307, December 2007.

[75] Patrick F. Tekavec, Thomas R. Dyke, and Andrew H. Marcus. Wave packet interferometry and quantum state reconstruction by acousto-optic phase modulation. The Journal of Chemical Physics, 125(19):194303, November 2006.

[76] Khadga J. Karki, Julia R. Widom, Joachim Seibt, Ian Moody, Mark C. Lonergan, TÃţnu Pullerits, and Andrew H. Marcus. Coherent two-dimensional photocurrent spectroscopy in a PbS quantum dot photocell. Nat Commun, 5, December 2014.

[77] D. L. Donoho. Compressed sensing. IEEE Trans. Inf. Theory, 52(4):1289 - 1306, 2006.

[78] Jacob N. Sanders, Semion K. Saikin, Sarah Mostame, Xavier Andrade, Julia R. Widom, Andrew H. Marcus, and Alán Aspuru-Guzik. Compressed Sensing for Multidimensional Spectroscopy Experiments. J. Phys. Chem. Lett., 3(18):2697-2702, September 2012.

[79] Alan D. Bristow, Tianhao Zhang, Mark E. Siemens, Steven T. Cundiff, and R. P. Mirin. Separating Homogeneous and Inhomogeneous Line Widths of Heavy- and Light-Hole Excitons in Weakly Disordered Semiconductor Quantum Wells. J. Phys. Chem. B, 115(18):5365-5371, May 2011.

[80] Lijun Yang, Igor V. Schweigert, Steven T. Cundiff, and Shaul Mukamel. Two-dimensional optical spectroscopy of excitons in semiconductor quantum wells: Liouville-space pathway analysis. Phys. Rev. B, 75(12):125302, March 2007.

[81] Xiaoqin Li, Tianhao Zhang, Camelia N. Borca, and Steven T. Cundiff. Many-Body Interactions in Semiconductors Probed by Optical Two-Dimensional Fourier Transform Spectroscopy. Phys. Rev. Lett., 96(5):057406, 2006.

[82] Daniel B. Turner, Patrick Wen, Dylan H. Arias, Keith A. Nelson, Hebin Li, Galan Moody, Mark E. Siemens, and Steven T. Cundiff. Persistent exciton-type many-body interactions in GaAs quantum wells measured using two-dimensional optical spectroscopy. Phys. Rev. B, 85(20):201303, 2012.

[83] Alan D. Bristow, Denis Karaiskaj, Xingcan Dai, Richard P. Mirin, and Steven T. Cundiff. Polarization dependence of semiconductor exciton and biexciton contributions to phase-resolved optical two-dimensional Fourier-transform spectra. Phys. Rev. B, 79(16):161305, 2009.

[84] Denis Karaiskaj, Alan D. Bristow, Lijun Yang, Xingcan Dai, Richard P. Mirin, Shaul Mukamel, and Steven T. Cundiff. Two-Quantum Many-Body Coherences in Two-Dimensional FourierTransform Spectra of Exciton Resonances in Semiconductor Quantum Wells. Phys. Rev. Lett., 104(11):117401, March 2010.

[85] Gaël Nardin, Galan Moody, Rohan Singh, Travis M. Autry, Hebin Li, François Morier-Genoud, and Steven T. Cundiff. Coherent Excitonic Coupling in an Asymmetric Double InGaAs Quantum Well Arises from Many-Body Effects. Phys. Rev. Lett., 112(4):046402, January 2014.

[86] Gaël Nardin, Rohan Singh, Travis M. Autry, Galan Moody, Hebin Li, François Morier-Genoud, and Steven T. Cundiff. Coupling in InGaAs Double Quantum Wells Studied with 2d Fourier Transform Spectroscopy. In CLEO: 2013, OSA Technical Digest (online), page QM2D.5. Optical Society of America, June 2013.

[87] A. J. Ramsay. A review of the coherent optical control of the exciton and spin states of semiconductor quantum dots. Semicond. Sci. Technol., 25(10):103001, October 2010.

[88] G. Moody, R. Singh, H. Li, I. A. Akimov, M. Bayer, D. Reuter, A. D. Wieck, A. S. Bracker, D. Gammon, and S. T. Cundiff. Influence of confinement on biexciton binding in semiconductor quantum dot ensembles measured with two-dimensional spectroscopy. Phys. Rev. B, 87(4):041304, January 2013.

[89] G. Moody, M. E. Siemens, A. D. Bristow, X. Dai, A. S. Bracker, D. Gammon, and S. T. Cundiff. 
Exciton relaxation and coupling dynamics in a GaAs/AlGaAs quantum well and quantum dot ensemble. Phys. Rev. B, 83(24):245316, 2011.

[90] G. Moody, R. Singh, H. Li, I.A. Akimov, M. Bayer, D. Reuter, A.D. Wieck, and S.T. Cundiff. Correlation and dephasing effects on the non-radiative coherence between bright excitons in an InAs QD ensemble measured with 2d spectroscopy. Solid State Communications, 163:65-69, 2013.

[91] Daniel B. Turner, Yasser Hassan, and Gregory D. Scholes. Exciton Superposition States in CdSe Nanocrystals Measured Using Broadband Two-Dimensional Electronic Spectroscopy. Nano Lett., 12(2):880-886, 2012.

[92] Graham B. Griffin, Sandrine Ithurria, Dmitriy S. Dolzhnikov, Alexander Linkin, Dmitri V. Talapin, and Gregory S. Engel. Two-dimensional electronic spectroscopy of CdSe nanoparticles at very low pulse power. The Journal of Chemical Physics, 138(1):014705-014705-11, January 2013.

[93] Bo Sun, Diogo B. Almeida, Rohan Singh, Geoffrey M. Diederich, Mark E. Siemens, Lazaro A. Padilha, Wan K. Bae, Jeffrey Pietryga, Victor Klimov, and Steven Cundiff. Two Dimensional Coherent Spectroscopy of CdSe/ZnS Colloidal Quantum Dots at Cryogenic Temperatures. In CLEO: 2015 (2015), paper JW2A.54, page JW2A.54. Optical Society of America, May 2015.

[94] B. Patton, W. Langbein, U. Woggon, L. Maingault, and H. Mariette. Time- and spectrallyresolved four-wave mixing in single CdTe/ZnTe quantum dots. Phys. Rev. B, 73(23):235354, 2006.

[95] Jacek Kasprzak and Wolfgang Langbein. Coherent response of individual weakly confined exciton-biexciton systems. J. Opt. Soc. Am. B, 29(7):1766-1771, 2012.

[96] C. Weisbuch, M. Nishioka, A. Ishikawa, and Y. Arakawa. Observation of the coupled excitonphoton mode splitting in a semiconductor quantum microcavity. Phys. Rev. Lett., 69(23):3314, 1992.

[97] T. K. Paraïso, M. Wouters, Y. Léger, F. Morier-Genoud, and B. Deveaud-Plédran. Multistability of a coherent spin ensemble in a semiconductor microcavity. Nat Mater, 9(8):655-660, August 2010.

[98] M. Vladimirova, S. Cronenberger, D. Scalbert, K. V. Kavokin, A. Miard, A. Lemaître, J. Bloch, D. Solnyshkov, G. Malpuech, and A. V. Kavokin. Polariton-polariton interaction constants in microcavities. Phys. Rev. B, 82(7):075301, 2010.

[99] N. Takemura, S. Trebaol, M. Wouters, M. T. Portella-Oberli, and B. Deveaud. Polaritonic Feshbach resonance. Nat Phys, 10(7):500-504, July 2014.

[100] Daniel B. Turner and Keith A. Nelson. Coherent measurements of high-order electronic correlations in quantum wells. Nature, 466(7310):1089-1092, August 2010.

[101] Brian L. Wilmer, Felix Passmann, Michael Gehl, Galina Khitrova, and Alan D. Bristow. Multidimensional coherent spectroscopy of a semiconductor microcavity. Phys. Rev. B, 91(20):201304, May 2015.

[102] Kin Fai Mak, Changgu Lee, James Hone, Jie Shan, and Tony F. Heinz. Atomically Thin MoS2: A New Direct-Gap Semiconductor. Phys. Rev. Lett., 105(13):136805, September 2010.

[103] Hualing Zeng, Junfeng Dai, Wang Yao, Di Xiao, and Xiaodong Cui. Valley polarization in MoS2 monolayers by optical pumping. Nat Nano, 7(8):490-493, August 2012.

[104] Kin Fai Mak, Keliang He, Jie Shan, and Tony F. Heinz. Control of valley polarization in monolayer MoS2 by optical helicity. Nat Nano, 7(8):494-498, August 2012.

[105] Diana Y. Qiu, Felipe H. da Jornada, and Steven G. Louie. Optical Spectrum of MoS2: ManyBody Effects and Diversity of Exciton States. Phys. Rev. Lett., 111(21):216805, November 2013.

[106] Yumeng You, Xiao-Xiao Zhang, Timothy C. Berkelbach, Mark S. Hybertsen, David R. Reichman, and Tony F. Heinz. Observation of biexcitons in monolayer WSe2. Nat Phys, 11(6):477-481, June 2015.

[107] Galan Moody, Chandriker Kavir Dass, Kai Hao, Chang-Hsiao Chen, Lain-Jong Li, Akshay Singh, 
Kha Tran, Genevieve Clark, Xiaodong Xu, Gunnar Bergauser, Ermin Malic, Andreas Knorr, and Xiaoqin Li. Intrinsic Exciton Linewidth in Monolayer Transition Metal Dichalcogenides. arXiv:1410.3143 [cond-mat], October 2014. arXiv: 1410.3143.

[108] Qing Hua Wang, Kourosh Kalantar-Zadeh, Andras Kis, Jonathan N. Coleman, and Michael S. Strano. Electronics and optoelectronics of two-dimensional transition metal dichalcogenides. Nat Nano, 7(11):699-712, November 2012.

[109] Jon A. Schuller, Sinan Karaveli, Theanne Schiros, Keliang He, Shyuan Yang, Ioannis Kymissis, Jie Shan, and Rashid Zia. Orientation of luminescent excitons in layered nanomaterials. Nat Nano, 8(4):271-276, April 2013.

[110] P. Dey, J. Paul, G. Moody, C. E. Stevens, N. Glikin, Z. D. Kovalyuk, Z. R. Kudrynskyi, A. H. Romero, A. Cantarero, D. J. Hilton, and D. Karaiskaj. Biexciton formation and exciton coherent coupling in layered GaSe. The Journal of Chemical Physics, 142(21):212422, June 2015.

[111] P. Dey, J. Paul, N. Glikin, Z. D. Kovalyuk, Z. R. Kudrynskyi, A. H. Romero, and D. Karaiskaj. Mechanism of excitonic dephasing in layered InSe crystals. Phys. Rev. B, 89(12):125128, March 2014.

[112] Franklin D. Fuller and Jennifer P. Ogilvie. Experimental Implementations of Two-Dimensional Fourier Transform Electronic Spectroscopy. Annual Review of Physical Chemistry, 66(1):667690, 2015.

[113] Oleksiy Roslyak and Shaul Mukamel. Multidimensional pump-probe spectroscopy with entangled twin-photon states. Phys. Rev. A, 79(6):063409, June 2009.

[114] Marten Richter and Shaul Mukamel. Ultrafast double-quantum-coherence spectroscopy of excitons with entangled photons. Phys. Rev. A, 82(1):013820, July 2010.

[115] M. G. Raymer, Andrew H. Marcus, Julia R. Widom, and Dashiell L. P. Vitullo. Entangled Photon-Pair Two-Dimensional Fluorescence Spectroscopy (EPP-2dfs). J. Phys. Chem. B, 117(49):15559-15575, December 2013.

[116] Konstantin E. Dorfman and Shaul Mukamel. Multidimensional spectroscopy with entangled light: loop vs ladder delay scanning protocols. New J. Phys., 16(3):033013, March 2014.

[117] Alejandro González-Tudela, Fabrice P. Laussy, Carlos Tejedor, Michael J. Hartmann, and Elena del Valle. Two-photon spectra of quantum emitters. New J. Phys., 15(3):033036, March 2013.

[118] Alejandro González-Tudela, Elena del Valle, and Fabrice P. Laussy. Optimization of photon correlations by frequency filtering. Phys. Rev. A, 91(4):043807, April 2015. 